\title{
A Review: Carbon Nanotube-Based Piezoresistive Strain Sensors
}

\author{
Waris Obitayo and Tao Liu \\ High-Performance Materials Institute, FAMU-FSU College of Engineering, Florida State University, 2525 Pottsdamer Street, \\ Tallahassee, FL 32310, USA \\ Correspondence should be addressed to Tao Liu, liutao@eng.fsu.edu
}

Received 15 December 2011; Accepted 2 February 2012

Academic Editor: Andrea Cusano

Copyright ( $) 2012$ W. Obitayo and T. Liu. This is an open access article distributed under the Creative Commons Attribution License, which permits unrestricted use, distribution, and reproduction in any medium, provided the original work is properly cited.

\begin{abstract}
The use of carbon nanotubes for piezoresistive strain sensors has acquired significant attention due to its unique electromechanical properties. In this comprehensive review paper, we discussed some important aspects of carbon nanotubes for strain sensing at both the nanoscale and macroscale. Carbon nanotubes undergo changes in their band structures when subjected to mechanical deformations. This phenomenon makes them applicable for strain sensing applications. This paper signifies the type of carbon nanotubes best suitable for piezoresistive strain sensors. The electrical resistivities of carbon nanotube thin film increase linearly with strain, making it an ideal material for a piezoresistive strain sensor. Carbon nanotube composite films, which are usually fabricated by mixing small amounts of single-walled or multiwalled carbon nanotubes with selected polymers, have shown promising characteristics of piezoresistive strain sensors. Studies also show that carbon nanotubes display a stable and predictable voltage response as a function of temperature.
\end{abstract}

\section{Introduction}

Carbon nanotubes have drawn much attention since their discovery in 1991 [1] because of their unique electronic and mechanical properties. Electronically, single-walled carbon nanotubes can be metallic, semiconducting, or small-gap semiconducting, depending on the graphene lattice orientation, with respect to the axis of the tube. They also have very interesting electromechanical properties [2] and could be useful in applications for piezoresistive strain sensors such as strain gauges.

The applications of strain sensors are mainly used in engineering fields for damage detection and characterization of structures. Strain measurements by the traditional sensors (metal and semiconductor strain gauges) have shown high sensitivities and are less expensive. But despite these advantages, there are drawbacks. They are fixed directional sensors, meaning strain can only be measured in a specific direction; they have low resolution at nanoscale and cannot be embedded in structural materials. The fiber Bragg grating (FBG) sensors also known as optical fiber sensors mostly used as trunk lines for the transmission of information have been attracting special interest recently due to their strain sensing characteristics [18]. In a manner similar to traditional strain gauges, calibration of displacement optical fiber is related to the strain resistivities of the fiber by appropriate gauge factors. FBG strain sensors match quite well with other composites materials like glass and carbon fiber composites, which are used in modern constructions. FGB strain sensors can be embedded into composites or can be fixed directly or as patches on the surface of the structural materials like normal strain gauges. They can measure very high strain $(>10,000 \mu \mathrm{m} / \mathrm{m})$ and are well suited to highly stressed composites constructions. The gauge factor is within the range of 0.77 to 0.81 [19]. One of the major disadvantages of FGB strain sensors is the insensitivity to strain on a nanoscale. Thin films piezoresistive strain sensors of single-walled and multiwalled carbon nanotubes serve as good alternatives for developing new sensors because of their outstanding properties. Carbon nanotube thin film strain sensors can be embedded into structural materials and operate as both multidirectional and multifunctional sensors with high strain resolution on nanoscale [20]. The electromechanical properties of carbon nanotube thin film strain sensors exhibit 


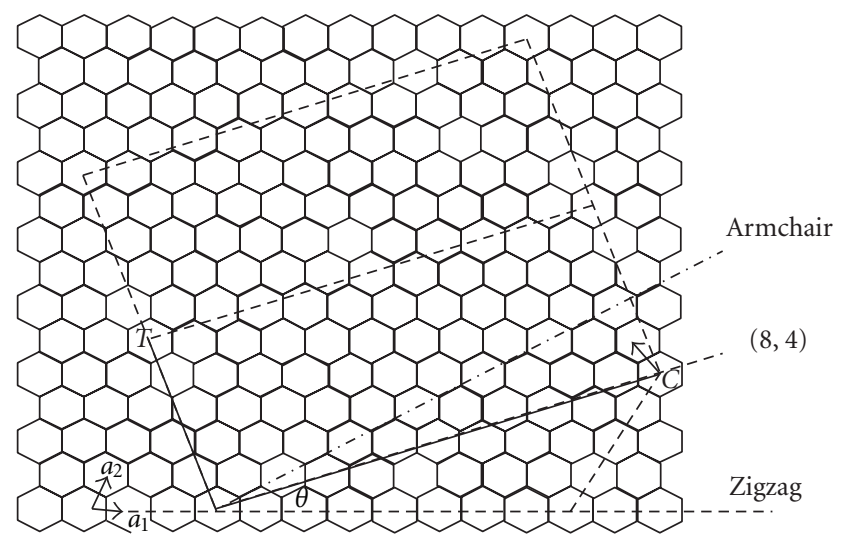

FIGURE 1: A carbon nanotube $(n, m)$ can be generated by rolling a graphene sheet along the chiral vector $C=n a_{1}+m a_{2}$ on the graphene sheet where $a_{1}$ and $a_{2}$ are graphene lattice vectors. The carbon nanotube can also be characterized by the diameter $d$ and the chiral angle $\theta$ with respect to the zig-zag axis, $\theta=0^{\circ}$. The diagram represents a construction of a $(8,4)$ single-walled carbon nanotube [3].

excellent characteristics compared to the traditional sensors due to a combination of high elastic moduli and outstanding electrical properties.

\section{Structure of Carbon Nanotubes}

The structure of carbon nanotubes has significant influence on its properties: electrical, mechanical, thermal, and optical conductivity. For example, the band gap of semiconducting single-walled carbon nanotubes is dependent on the diameter. An increase in diameter leads to a decrease in the band gap [4].

2.1. Single-Walled Carbon Nanotubes. A single-walled carbon nanotube can be seen as a seamless cylinder achieved by rolling up a section of a graphene sheet. Its structure is uniquely described by a roll-up vector $C=n a_{1}+m a_{2}$, where the $n$ and $m$ are the chiral indices and $a_{1}$ and $a_{2}$ are the graphene lattice vectors. The unit cell of a single-walled carbon nanotube is defined by the chiral indices, $n$ and $m$, which is the area covered by the translational vectors $T$ and $C$. The magnitude of the translational vector $T$ is along the axis of the single-walled carbon nanotube and perpendicular to the $C$ vector as shown in Figure 1.

The diameter $d$ and the chiral angle $\theta$ can be determined once the chiral indices $n$ and $m$ are known: $d=$ $|C| / \pi=(a / \pi)\left(n^{2}+n m+m^{2}\right)^{1 / 2}$ and $\cos \theta=(2 n+m) /$ $2 \sqrt{n^{2}+n m+m^{2}}$, where $a=\sqrt{3} a_{c c}=2.46 \AA$ is the lattice constant and $a_{c c}=1.42 \AA$ is the bond distance of the graphene.

2.2. Multiwalled Carbon Nanotubes. A multiwalled carbon nanotube is made of multiple rolled layers of graphene sheets with inner diameters as small as those of the single-walled carbon nanotubes, which can be up to tens of nanometers. The outer diameters vary from a few nanometers to tens of nanometers. Multiwalled carbon nanotubes have an inter- layer spacing of approximately $0.34 \mathrm{~nm}$, which is slightly greater than graphite $(0.335 \mathrm{~nm})$. This difference is attributed to van der Waals forces between consecutive cylinders [1].

\section{Properties of Carbon Nanotubes}

3.1. Mechanical Properties. The $\sigma$ bonds and the tubular structure of carbon nanotubes are responsible for its excellent mechanical properties. Many analytical calculations have been used to estimate the Young's modulus of single-walled carbon nanotubes to be least $1 \mathrm{TPa}[21,22]$. The experiments of Wong et al. corresponded with previous experimental results indicating a mean value of $1.28 \pm 0.5 \mathrm{TPa}$ for multiwalled carbon nanotubes [23]. Carbon nanotubes have also shown high tensile strength of up to $40 \%$ without any form of plastic deformation, brittle-like behavior, or rupture. This is due to the extra energy absorption required for the hollow structures of carbon nanotubes compared to most materials. The tensile strength is said to be on the order of a hundred $\mathrm{GPa}$, which is approximately a thousand times harder than steel [24].

These superior mechanical properties make carbon nanotubes suitable as reinforcing materials in composites. Andrews et al. [25] showed that by adding 5\% of singlewalled carbon nanotubes, the tensile strength and Young's modulus of the composite increases by $90 \%$ and $150 \%$, respectively. The excellent elastic property of carbon nanotubes allows them to store a huge quantity of energy.

3.2. Electronic Properties. A carbon nanotube having chirality with $n=m$ tubes with no band gap is metallic. It is semiconducting when $n \neq m$ with some band gap. The subset with $n-m=3 q$ where $q$ is an integer shows a small band gap induced by curvature of the graphene sheet and is called semimetallic, quasimetallic, or small-gap semiconducting. The band gaps of both semiconducting and small-gap semiconducting single-walled carbon nanotubes decrease with $1 / r_{t}$ and $1 / r_{t}^{2}$, respectively, (as shown in Figure 2). The smallgap semiconducting carbon nanotubes formed due to curvature effects are usually considered metallic at room temperature for experimental conditions [4].

The electronic structure of multiwalled carbon nanotubes is more complicated due to the various coaxially arranged single-walled carbon nanotubes. Electron transport in multiwalled carbon nanotubes is said to be similar to that of the larger diameter single-walled carbon nanotubes because most of the electric current passing through a multiwalled carbon nanotubes from the outside is mostly confined to the outermost cylindrical layer [26, 27].

The outstanding electronic properties of carbon nanotubes make them useful in electronic devices and sensor applications.

3.3. Electromechanical Properties. The electronic property and structure relationship imply that mechanical deformation can alter the band structure of carbon nanotubes. This effect can be useful in the application of carbon nanotubes as strain sensors. 


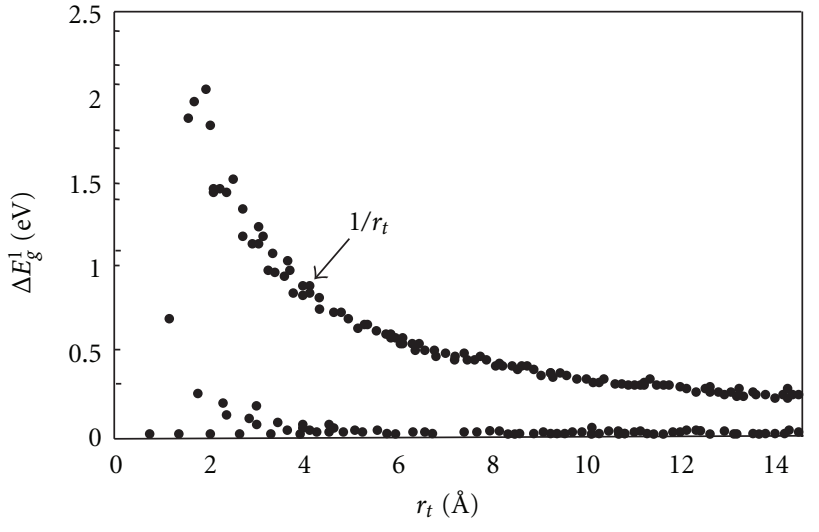

(a)

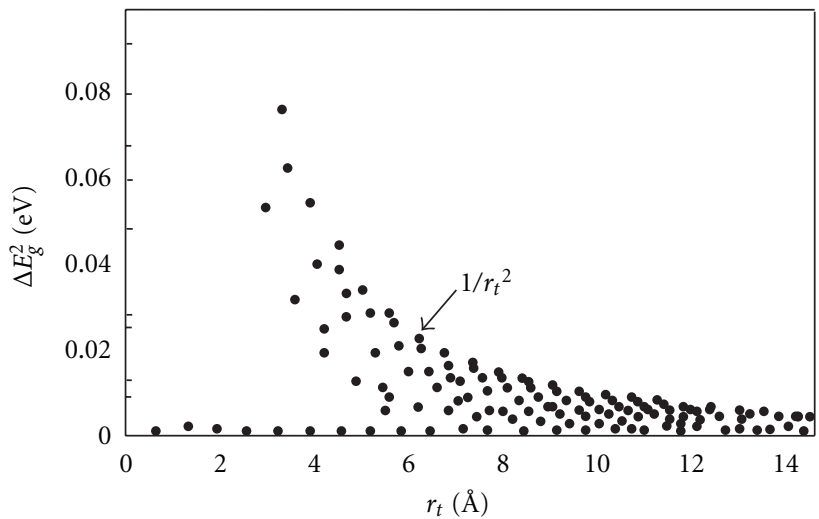

(b)

FIgURE 2: The primary gap $\Delta E_{g}^{1}$ (semiconducting single-walled carbon nanotubes) scales as $1 / r$ (a), while for the curvature-induced band gap $\Delta E_{g}^{2}$ (small-gap semiconducting carbon nanotubes) scales as $1 / r^{2}$. At $\Delta E_{g}^{1}=\Delta E_{g}^{2}=0$ relates to armchair nanotubes, which maintain their metallic character [4].

The electronic structure of a carbon nanotube takes into account the periodic boundary condition caused by rolling a graphene sheet into a cylindrical shape. This effect results in the quantization of the electron wave functions within the tube. The quantization effect cuts in the direction of electrons along the tube's axis. The bond length of the carbon nanotube is altered when strain is applied by shifting the periodicity of the quantization, resulting in a change in the conic section, which decreases or increases the band gap.

The unstrained state of a metallic carbon nanotube shows no band gap, while the band gap of a semiconducting carbon nanotube varies as an inverse to the diameter

$$
E_{\text {Gap }}^{0}=\frac{2 \gamma a}{\sqrt{3} d} .
$$

(See [4].) $E_{\text {Gap }}^{0}$ is $\sim 0.1$ to $2 \mathrm{eV}$ for an average semiconducting carbon nanotube, the tight binding overlap integral $\gamma$ is $2.6 \mathrm{eV}, d$ is the diameter of the carbon nanotube, and $a$ is the unit vector of graphene.

Several models have been used to illustrate the electronic properties of carbon nanotubes under strain (both analytical and computational methods). Yang et al. [28] reported the effects of strain $(\varepsilon)$ and torsion $(\xi)$ on the change in band gap of semiconducting and metallic carbon nanotubes

$$
\Delta E_{g}=\operatorname{sgn}(2 p+1) 3 \gamma[(1+v) \varepsilon \cos 3 \theta]+\xi \sin 3 \theta,
$$

where $v$ is the Poisson's ratio of the tube $\sim 0.20$ and $\theta$ is the chiral angle. The sign of the equation depends on $p$, where $n-m=3 q+p$, where $q$ is an integer and $p=+1,0,-1$. The bandgap of metallic carbon nanotubes $(n \neq m)$ belonging to the class $p=0$ increases with strain, while semiconducting carbon nanotube of class $p=+1$ also shows an increase in band gap with strain. The semiconducting carbon nanotubes of class $p=-1$ shows a decrease in band gap with strain. A maximum band gap change of $d E_{g} / d \varepsilon \sim 95 \mathrm{meV} / \%$ is predicted from (1). This equation works well for larger diameters $(>1 \mathrm{~nm})$ [7] of semiconducting and metallic carbon nanotubes. Kleiner and Eggert [29] took into account the smaller diameter carbon nanotubes in which the curvature effect significantly affects the change in band gap with strain. The band gap of small-gap semiconducting carbon nanotubes as a function of chirality and deformation can be expressed as

$$
\begin{aligned}
\Delta E_{g}= & -\operatorname{sgn}\left[\frac{\gamma a^{2}}{4 d^{2}}-\frac{a b \sqrt{3}}{2} \varepsilon\right] \cos 3 \theta \\
& -\frac{a b \sqrt{3}}{2} \xi \sin 3 \theta,
\end{aligned}
$$

where $b(\sim 3.5 \mathrm{eV} / \AA)$ is the linear change in transfer integral with change in bond length. The equation predicts a maximum band gap change of $d E_{g} / d \varepsilon \sim 75 \mathrm{meV} / \%$ [29].

Strain can induce a change in the electrical resistance of carbon nanotubes, and this change can be calculated based on the modification of the band gap. A thermally activated transport model can be used to relate the measured resistance to the change in band gap in the carbon nanotube [7]

$$
R=R^{C}+\frac{1}{|t|^{2}} \frac{h}{8 e^{2}}\left(1+\exp \left(\frac{E_{\mathrm{Gap}}^{0}+\left(d E_{\mathrm{gap}} / d \varepsilon\right) \varepsilon}{K T}\right)\right) .
$$

$R^{C}$ is the contact resistance; $|t|$ is the average transmission coefficient; $h$ is Plank's constant; $e$ is the electron's quantity of the electricity; $K$ is the Boltzmann constant; $T$ is the absolute temperature; $d E_{\mathrm{gap}} / d \varepsilon$ is the strain-dependent band gap.

The value for the unstrained band gap of semiconducting carbon nanotubes is

$$
E_{\text {Gap }}^{0}=\frac{2 \gamma_{0} a}{\sqrt{3} d}
$$

(see [4],) and for small-gap semiconducting carbon nanotubes

$$
E_{\text {Gap }}^{0}=\frac{\gamma_{0} a^{2}}{4 d^{2}} .
$$




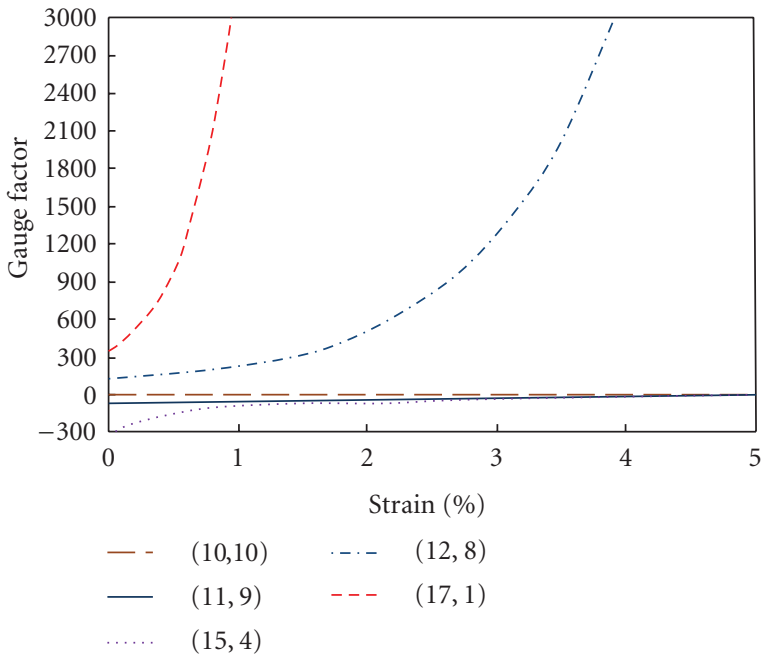

FIgURE 3: Gauge factor versus strain for carbon nanotubes with diamter $1.38 \mathrm{~nm}$ [5].

(See [30].) The piezoresistive sensing property is defined by the gauge factor $\left(G_{F}\right)$, which is the change in resistance divided by the strain.

$$
G_{F}=\frac{\Delta R}{R} \frac{1}{\varepsilon} .
$$

Substitute (4) into (8) and since $R^{C} \ll R$,

$$
G_{F}=\frac{\left(d E_{\mathrm{Gap}} / d \varepsilon\right) \exp \left(\left(E_{\mathrm{Gap}}^{0}+\left(d E_{\mathrm{gap}} / d \varepsilon\right) \varepsilon\right) / K T\right)}{K T\left[\exp \left(\left(E_{\mathrm{Gap}}^{0}+\left(d E_{\mathrm{gap}} / d \varepsilon\right) \varepsilon_{0}\right) / K T\right)\right]+1} .
$$

(See [31].) $\varepsilon_{0}$ is the pretension strain. The gauge factors of different types of carbon nanotubes can be calculated by inserting (2) and (5) for semiconducting carbon nanotubes and (3) and (6) for small-gap semiconducting carbon nanotubes into (8).

It can be seen from the equations that the maximum magnitude of gauge factor occurs for zig-zag $(n, 0)$ carbon nanotubes and the gauge factor decreases to a value of zero for armchair $(n, n)$ carbon nanotubes as the chiral angle increases as shown in Figure 3.

Carbon nanotube-based piezoresistive strain sensors have been shown to render high performance in their applications with a gauge factor greater than 2900 [32] compared to silicon-based sensors with a gauge factor of $\sim 200$ [33]. Tombler et al. [6] used an AFM tip to deform a metallic carbon nanotube suspended across a $600 \mathrm{~nm}$ trench in a silicon oxide substrate, while the current is measured through the tube. The experiment showed that the conductance of metallic carbon nanotube is reduced by two orders of magnitude at a strain of $\sim 3 \%$ as shown in Figure 4 . This phenomenon was unusual since a metallic carbon nanotubes show little or no response to deformation $[28,34,35]$. Simulation results showed that the decrease in conductance was due to the hybridization of $s p^{2}$ to $s p^{3}$ bonding at the carbon nanotube/ AFM tip contact. This effect caused an increase in the $\sigma$-electrons, and a decrease in the $\pi$-electrons which resulted in a decline in conductance $[2,6,36]$.
A similar experiment was also done by Minot et al. [7]. They measured the initial bandgap $\left(E_{g}\right)$ and the change in band gap $\left(d E_{g} / d \varepsilon\right)$ of suspended doubly clamped carbon nanotubes in a field effect transistor configuration. They differentiated between the metallic, small-gap semiconducting, and semiconducting carbon nanotubes by using the AFM tip as a local gate to tune the conductivity. The results from the seven carbon nanotubes used for the experiment showed that two of the semiconducting carbon nanotubes increased in conductance, one semiconducting, and two metallic carbon nanotubes decreased in conductance, and the conductance of the remaining two metallic carbon nanotubes was unaffected. It was concluded that the metallic carbon nanotubes with no change in conductance were metallic carbon nanotubes $(n=m)$, while the metallic carbon nanotubes with decreased in conductance were small-gap semiconducting carbon nanotubes. The semiconducting carbon nanotubes showed both an increase and a decrease in band gap as shown in Figure 5.

The strain-induced band gap of carbon nanotube field effect transistors on a thin film membrane was investigated by Grow et al. [8]. They examined the piezoresistive of carbon nanotubes under small strain. Carbon nanotubes were grown on a deformable membrane of silicon nitride and strained with gas pressure as shown in Figure 6. The conductance changes under strain of up to $0.2 \%$ for semiconducting and small-gap semiconducting carbon nanotubes. The majority of the small-gap semiconducting carbon nanotubes showed a decrease and an increase in conductance, while for the four semiconducting carbon nanotubes, three increases and one decreases as shown in Figure 7. The change in conductance was attributed to a change in band gap rather than the contact because the off-current was more than that of the on-current. The calculated band gaps were different from the predicted results, and for small-gap semiconducting carbon nanotubes, the band gap was more than expected for the diameters measured with atomic force microscopy (AFM). This inconsistency was attributed to the silicon nitrate substrate interaction. The increase and decrease exhibited by the small-gap semiconducting carbon nanotubes were likely attributed to the rough surface of the membrane by twisting and flattening itself over obstacles. This resulted in a local disordered structure of the carbon nanotubes. Distortion induced by strain, torsion, and substrate interaction can result in a rise in the band gap of metallic and small-gap semiconducting carbon nanotubes. The band gap of unstrained small-gap semiconducting carbon nanotubes was increased as well as intrinsically metallic carbon nanotubes. Due to the effect of the rough substrate on the electromechanical properties of carbon nanotubes, a smoother surface substrate is preferable for reproducibility in strain sensor applications.

Cao et al. [9] differentiated metallic, semiconducting, and small-gap semiconducting carbon nanotubes as shown in Figure 8. They calculated the gauge factor for small-gap semiconducting carbon nanotubes to be around 600 to 1000 for strains up to $0.2 \%$ (compared to silicon-based sensors $\sim 80$ to 200) [27]. For semiconducting carbon nanotubes, it was around 150 for strain below $0.15 \%$. A higher response 

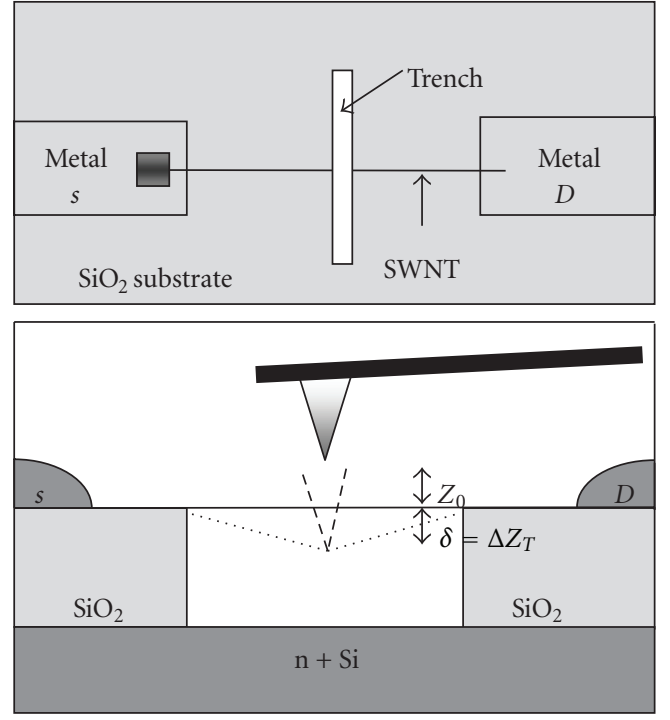

(a)

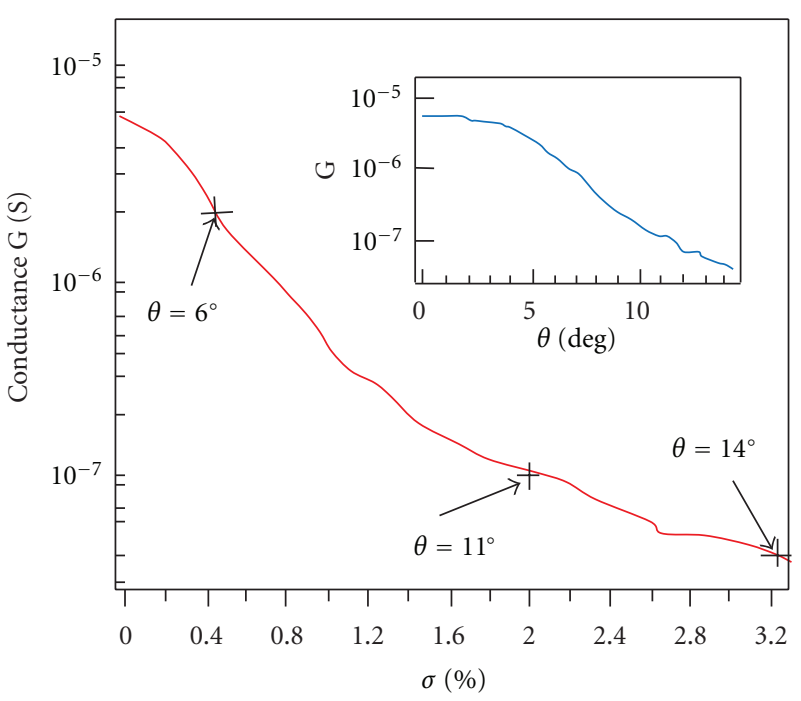

(b)

FIgURE 4: (a) A setup of Tombler's experiment: (top) top view of the device and (bottom) side view of the atomic force microscopy (AFM) pushing experiment. (b) The conductance versus mechanical deformation. Insert: conductance as a function of the deflection angle $\theta$ [6].

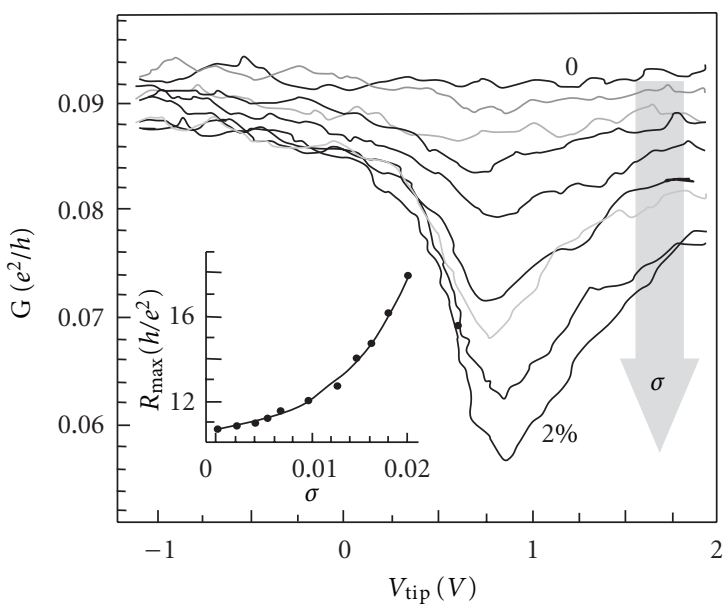

(a)

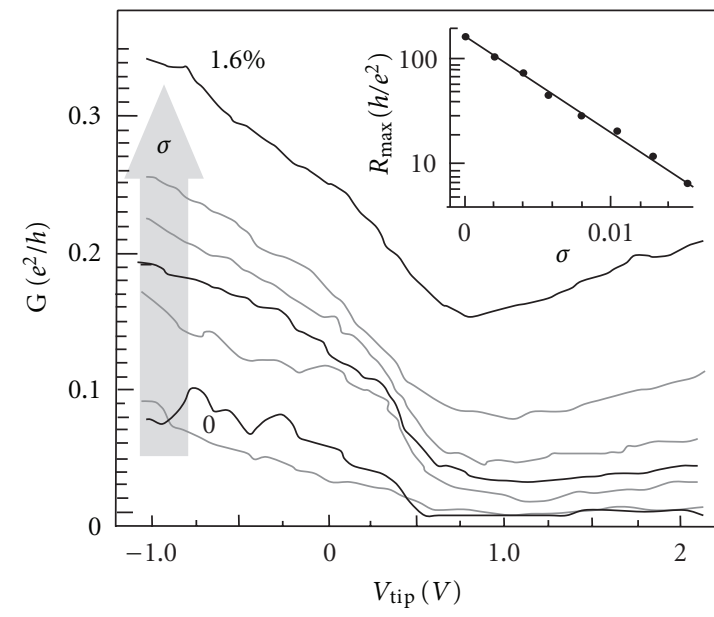

(b)

Figure 5: (a) $I-V_{g}$ versus strain. (a) The plot shows conductance decrease with strain for metallic single-walled carbon nanotubes. Insert: resistance in the OFF state versus strain. (b) Same as (a) for semiconducting single-walled carbon nanotubes shows an increase in conductance with strain [7].

was found for the three types of carbon nanotubes contradicting theoretical prediction. This discrepancy may be attributed to local deformation at the cantilever and platform edges. The experiments show that an increase and a decrease of conductance was found for semiconducting carbon nanotubes. Metallic carbon nanotubes were expected to show little or no response in conductance (according to theoretical prediction), but experimental results show a higher response. The small-gap semiconducting carbon nanotubes showed a decrease in conductance for both large and small strains, which differ from the theoretical prediction that conductance should decrease for strain $<0.2 \%$ and increase afterward. The variation between the experiment and theory may be due to local deformation, noise, or thermal effects, that is, between metallic and small-gap semiconducting carbon nanotubes.

\section{Resistance Properties of a Carbon Nanotube Network}

The strain sensing phenomenon in a carbon nanotube network is attributed to two types of resistances. The first is the intrinsic resistance, $R^{\mathrm{CNT}}$, and the second is the intertube resistances. The intertube resistances are divided into two types: the contact resistance, $R^{C}$, (resistance between tubes that are physically in contact) and the tunneling resistance, 

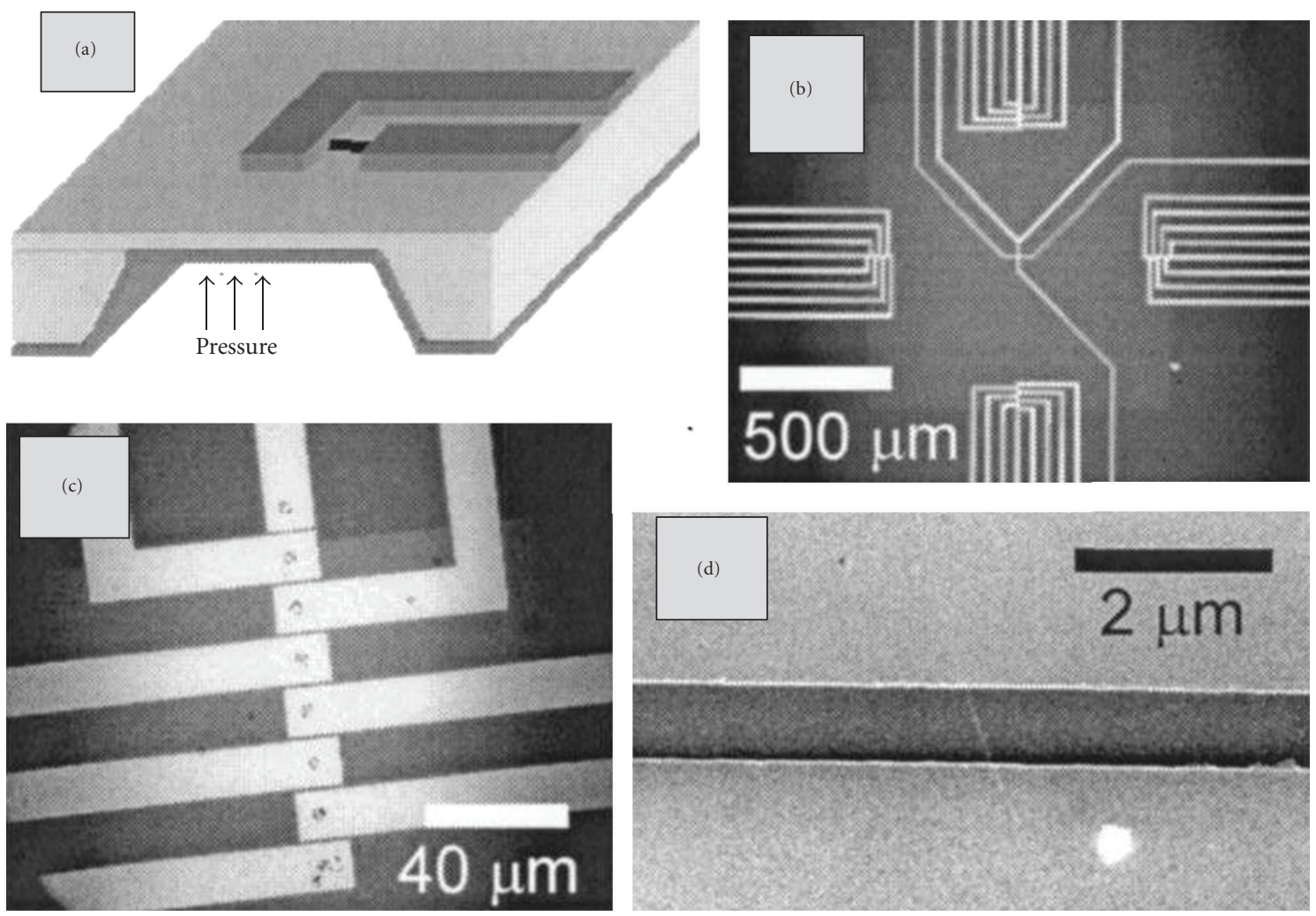

FIGURE 6: (a) A single-walled carbon nanotube device on a membrane with the bottom layer serving as the gate electrode. (b) An optical image of the membrane $(\sim 1 \mathrm{~mm}$ on a side) with electrodes. (c) The devices ( $14 \mu \mathrm{m}$ apart) are zoomed near the edge of the membrane, (d) SEM image of a single-walled carbon nanotube crossing the gap $(\sim 800 \mathrm{~nm})$ between two electrodes [8].

$R^{T}$, (resistance between tubes that are separated by small gap) as shown in Figure 9. The piezoresistive effects of a carbon nanotube network are primarily due to the modification in intrinsic resistance and intertube resistance.

4.1. Intrinsic Resistance of Carbon Nanotubes. Carbon nanotubes can act as good conductors because of their onedimensional structures, which allow electronic transport to occur ballistically $[26,37]$. Multiwalled carbon nanotubes have been found to have an intrinsic resistance of $0.2-$ $0.4 \mathrm{k} \Omega \mathrm{s} / \mu \mathrm{m}[38,39]$. The intrinsic resistance is subjected to modification under strain. Studies showed that the intrinsic resistance of single-walled carbon nanotubes increased considerably at a relatively small strain $[2,6,28,40,41]$. The intrinsic resistance of carbon nanotubes increases exponentially with the applied strain [7] and the extent to which it increases depends on the chiral angle of the carbon nanotubes as shown in Figure 10(a).

4.2. Intertube Resistance of Carbon Nanotubes. Despite the intrinsically high conductivity of carbon nanotubes, conduction in a carbon nanotube network is not correspondingly efficient. This is because intertube resistance is quite higher compared to the intrinsic resistance. The intertube resistance is made of two types as illustrated schematically in Figure 9. The first is contact resistance $R^{C}$, which is the resistance between carbon nanotubes that are physically in contact, such that conduction takes place between these carbon nanotubes through electron diffusion. The contact resistance has been shown to depend greatly on the contact region and has large values varying from a few hundreds to a thousand $\mathrm{k} \Omega$ s. These values depend on factors like arrangement of molecules across the interface and extent of the interfacial surface [28].

When there is a gap between the carbon nanotubes, conduction occurs by a tunneling mechanism [42, 43]. Tunneling resistance is expressed by the equation below [42]

$$
R^{T}=R_{0} e^{\lambda s},
$$

where $R^{T}$ is the tunneling resistance and assuming a small bias voltage and rectangular barrier:

$$
\begin{aligned}
R_{0} & =\frac{1}{C_{1}} \frac{S}{\sqrt{K}}, \\
\lambda & =C_{2} \sqrt{K} .
\end{aligned}
$$

The constants $C_{1}$ and $C_{2}$ are $3.16 \times 10^{10}$ and 1.0125 , respectively. $K$ is equal to the average height of the potential barrier $(\mathrm{eV})$ and $S$ is the gap $(\AA)$.

The intertube resistances can be modified through strain by widening the gap between the carbon nanotubes, which increases the resistance as shown in Figure 10(b). The effect of strain on the intertube resistance is less severe than that of the intrinsic resistance. 


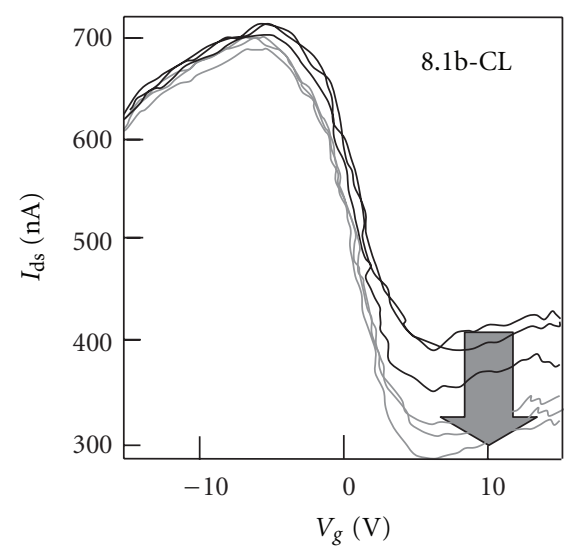

(a)

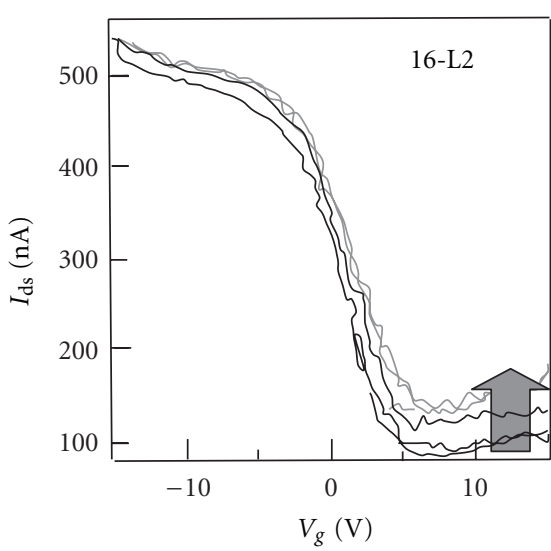

(b)

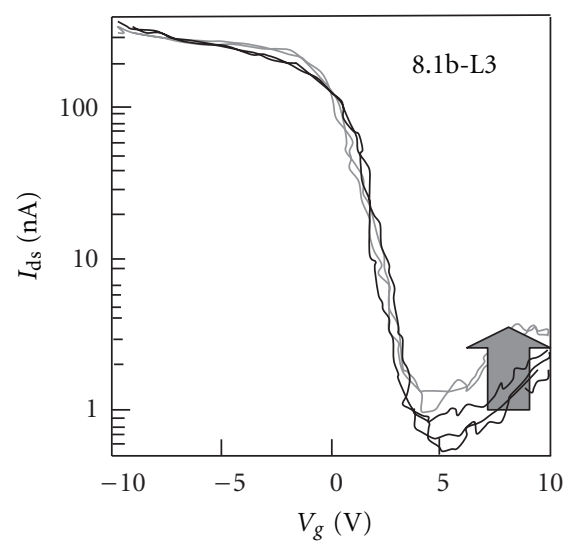

(c)

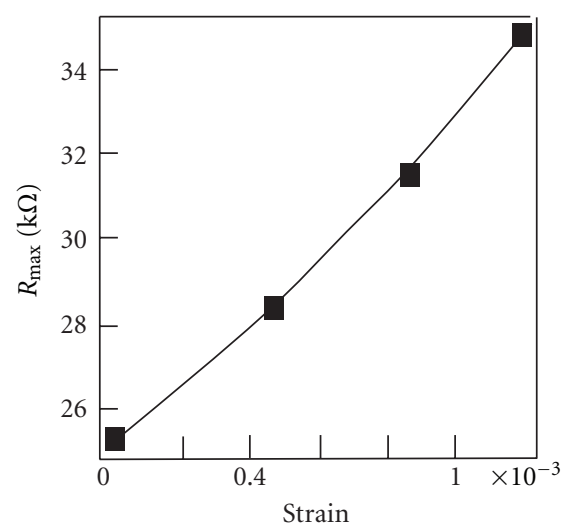

(d)

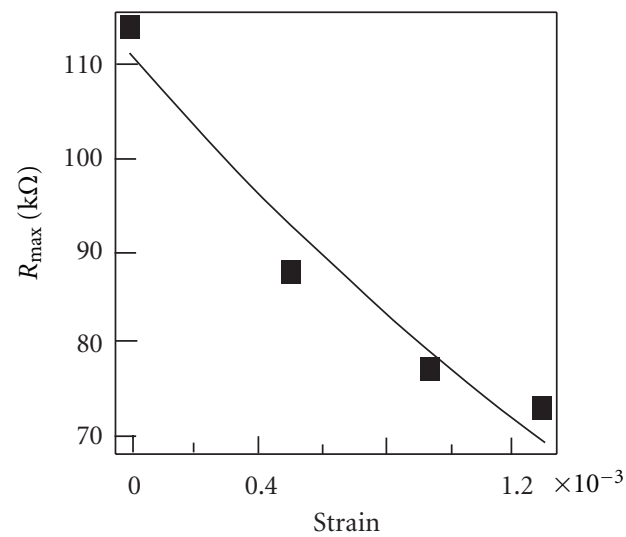

(e)

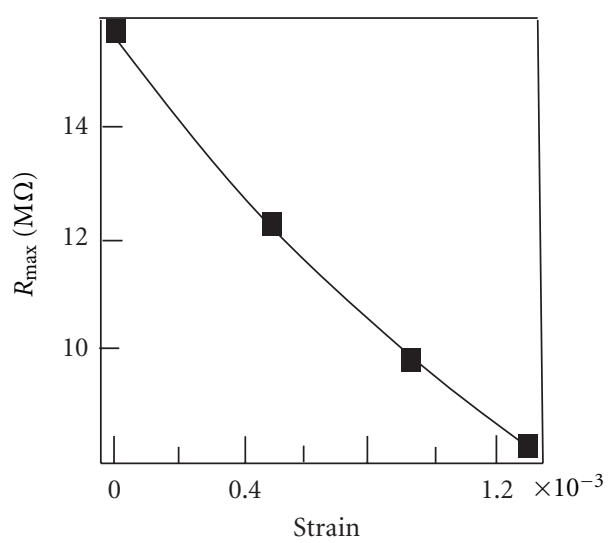

(f)

Figure 7: $(\mathrm{a}-\mathrm{c}) I_{\mathrm{ds}}-V_{g}$ curves for single-walled carbon nanotube devices on the membrane at $0,5,10,15$, and 0 psi $\left(V_{\mathrm{ds}}=10 \mathrm{mV}\right)$. The arrows indicate the direction of the curves with increasing pressure. The first two devices are for small-gap semiconducting carbon nanotubes, and the third one is for semiconducting carbon nanotubes. (d-f) $R_{\max }$ values of the $I_{\mathrm{ds}}-V_{g}$ curves versus strain and a fit to an exponential [8].

\section{Carbon Nanotube-Based Composites and Thin Films for Strain Sensing}

The strain sensing effectiveness of individual carbon nanotubes at the nanoscale is used to develop macroscale strain sensors. The use of individual carbon nanotubes have shown prominent accomplishment in sensor applications, but are faced with scaling to any realistic type of system, that is, difficulties in drawing significant current output and problems arising with methods of producing homogeneous carbon nanotubes with orientation and special locations. Thin films of carbon nanotubes avoid these difficulties. They have high current output and are not sensitive to spatial locations or orientation of individual carbon nanotubes [44]. Aligned carbon nanotubes in thin film networks display properties almost the same as individual carbon nanotubes [20]. 


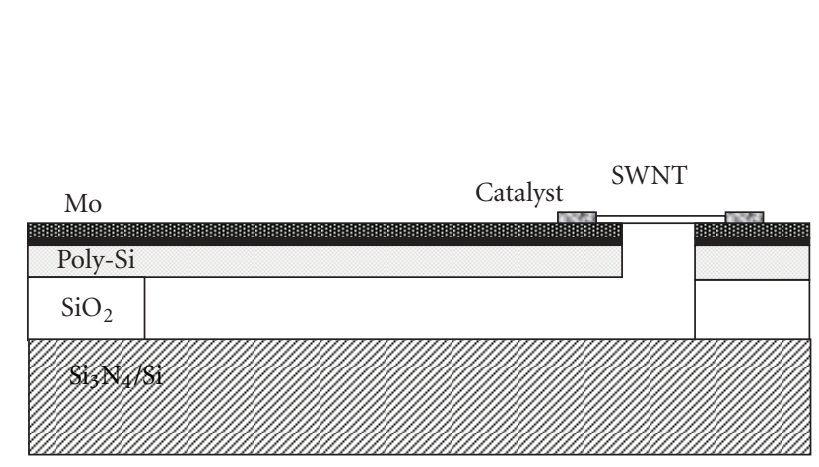

(a)

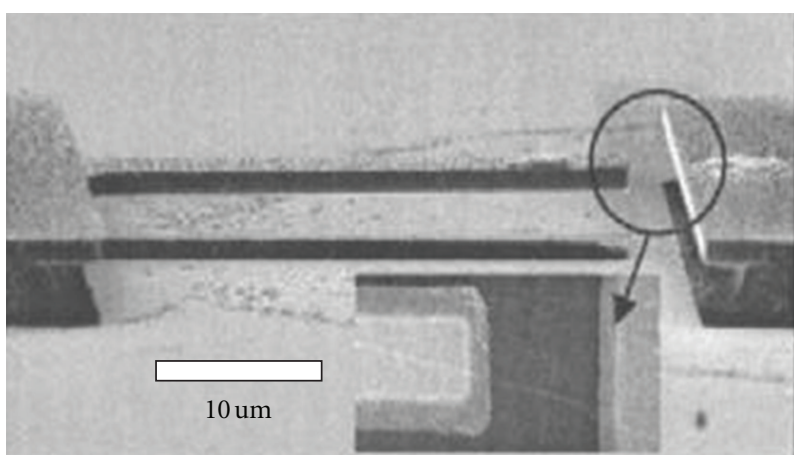

(b)

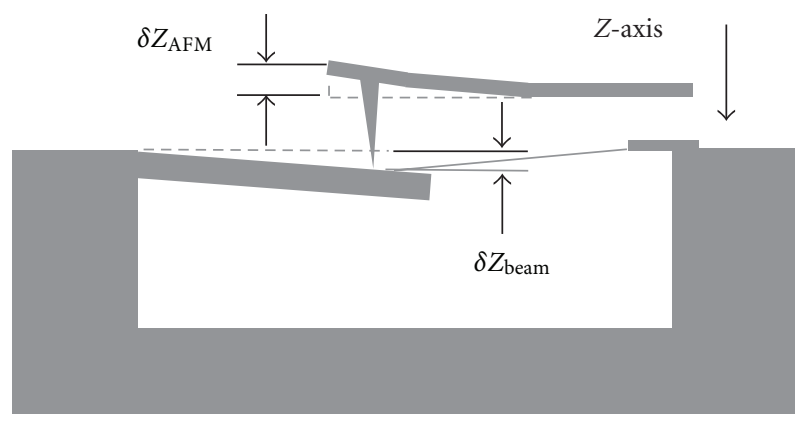

(c)

Figure 8: (a) A cantilever and a solid platform with a suspended nanotube between them. (b) An SEM image of the device. Insert: zoom-in view of a suspended single carbon nanotube. (c) Scheme for stretching a single-walled carbon nanotube [9].
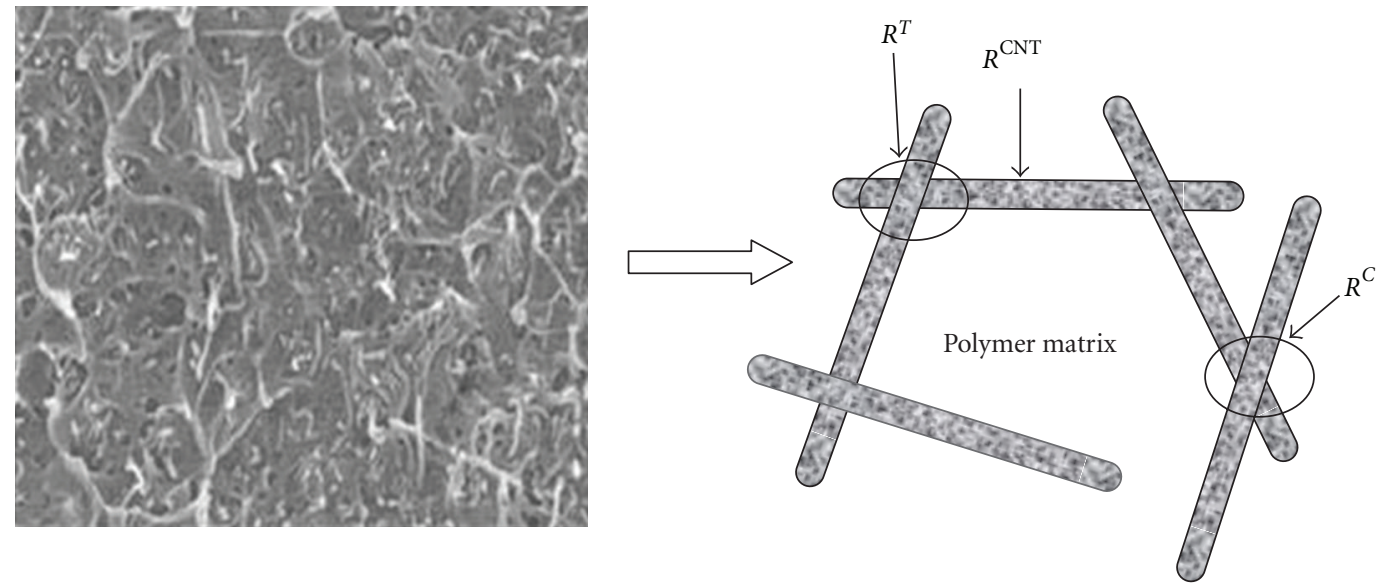

FIGURE 9: Illustration of carbon nanotubes network in a polymer matrix [10].

The use of single-walled carbon nanotube thin film strain sensors is restricted by limited control of purity, chirality, and electrical properties, but multiwalled carbon nanotubes are more economical and can be grown at relatively high purity. They are subjected to chirality-related restrictions on electrical properties and can be subjected to aggressive processing.

The strain sensing of carbon nanotube-polymer composites is mainly due to the piezoresistive properties of the conductive network of carbon nanotubes in the polymer matrices. When the network of carbon nanotubes is altered from mechanical deformation; there is an increase in the resistivity. This difference in the resistivity is associated with the modification of contact arrangements and the tunneling distance between carbon nanotubes.

Early studies were based on electromechanical sensing of conductive carbon nanotubes networks also known as "buckypapers." The buckypapers are usually fabricated by dispersing carbon nanotubes in a solvent using a surfactant. The carbon nanotubes solution is filtered, deposited, and dried on a substrate $[20,45]$. The resistivity of the carbon nanotube sheet can be measured by loading the substrate 


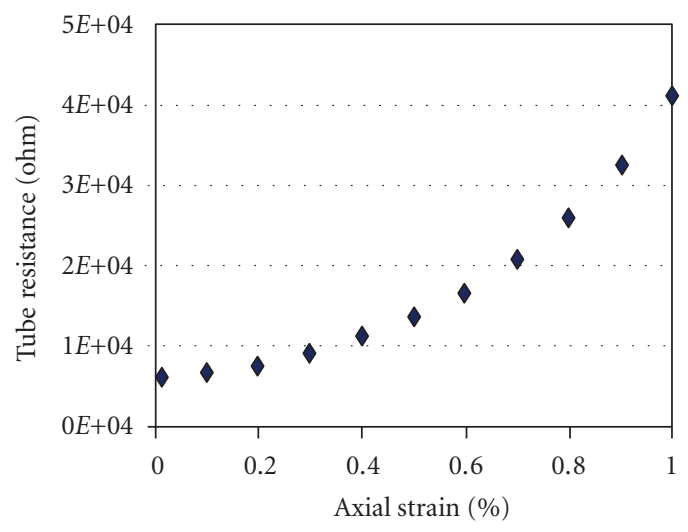

(a)

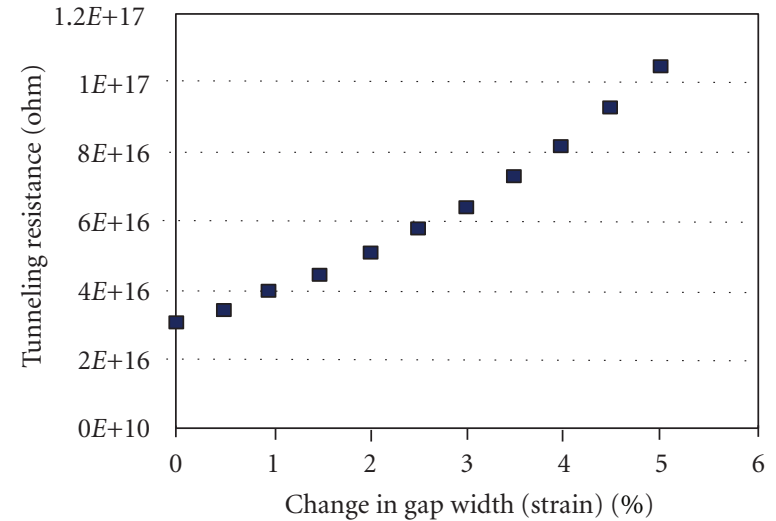

(b)

FigURE 10: The effect of strain on carbon nanotubes (a) intrinsic resistance of carbon nanotubes (b) intertube tunneling resistance of carbon nanotubes [10].

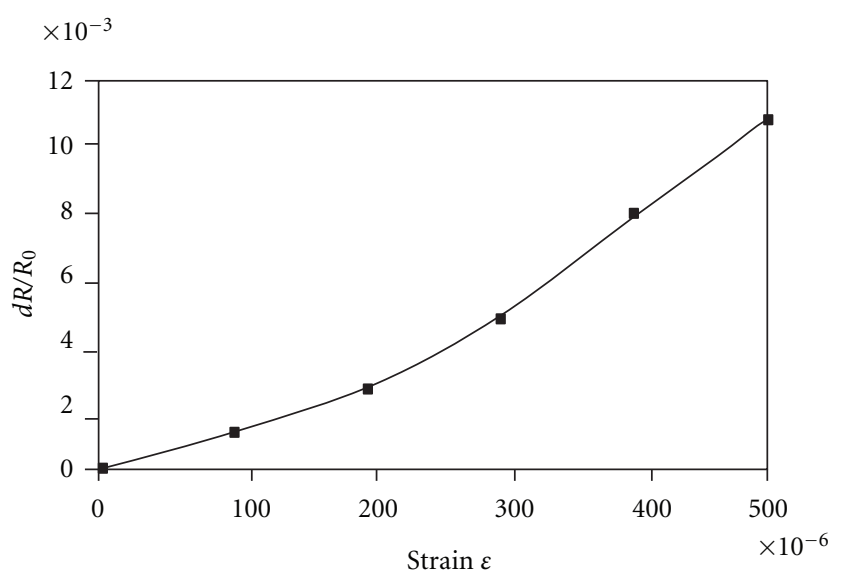

$\rightarrow-19^{\circ} \mathrm{C}$

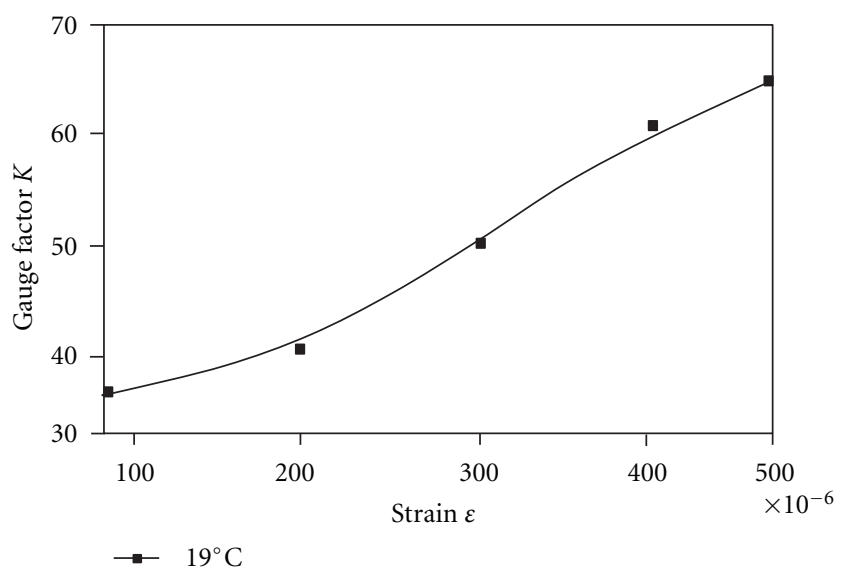

(b)

FIGURE 11: (a) The relative change in electrical resistance versus mechanical strain. (b) The gauge factor versus the mechanical strain for pristine single-walled carbon nanotubes. These values were measured at temperature $19^{\circ} \mathrm{C}[11]$.

under tension or compression. Analysis has shown that resistivity increases linearly under tension and decreases linearly under compression.

Li et al. [11] illustrate the piezoresistive effect of pristine carbon nanotube thin films using a three-point bending test as shown in Figure 11. The gauge factor was found to be 65 under normal temperature for strain up to 500 microstrains, but increases with temperature. The basis of the piezoresistive properties of carbon nanotube thin films was attributed to a change in the band gap caused by the strain [6]. Defects also play an important role in higher gauge factors [22, 46].

Wang et al. [12] showed how strain caused changes in the resistance of two types of carbon nanotubes. The piezoressitive properties of iodine-doped and iodine-undoped carbon nanotube films were studied using a three-point bending test. Gauge factors of about 125 and 65 were found for iodinedoped and iodine-undoped carbon nanotubes films less than 500 microstrains, respectively, as shown in Figure 12.
Different factors were associated with the piezoressitive properties of these two types of carbon nanotubes, that is, intertube contact resistance for the undoped carbon nanotubes and strain-induced band gap change for the doped carbon nanotubes.

Dharap et al. [45] used the strain sensing features of single-walled carbon nanotubes to develop a thin film that can be used on a macroscale. Experimental results showed that there was a linear change in voltage across the film when subjected to both tensional and compressive deformation. A movable four-point probe in contact with the single-walled carbon nanotubes thin film was used to measure the change in voltage and resistivity [47]

$$
\rho_{s}=\frac{V}{I} C\left(\frac{a}{d}, \frac{d}{s}\right)
$$

where $V$ is the voltage across the inner two probes, $I$ is the current across the outer two probes, and $C(a / d, d / s)$ depends 


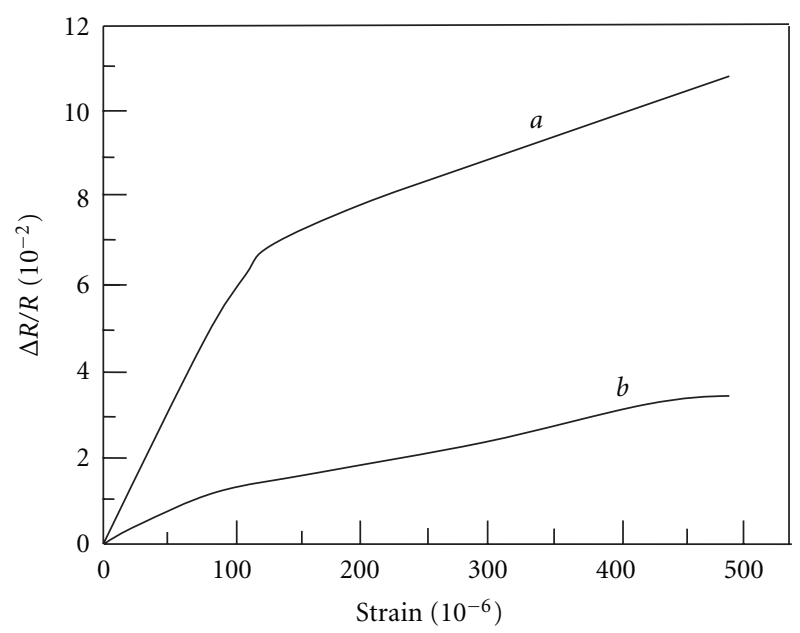

FIGURE 12: Relationship between the relative changes in the resistance of the carbon nanotubes as a function of tensile strain. (a) Iodine-doped and (b) iodine-undoped carbon nanotube film [12].

on the dimensions of the film ( $a, d, s$ are the length, width, and distance between the two probes in a four-point probe, resp.).

They concluded that the change in voltage was essentially due to a change in resistivity and not dimensions of the film. A constant resistivity was assumed for change in strain from $0 \%$ to $0.04 \%$

$$
\begin{gathered}
\rho_{s}=\frac{V_{1}}{I} C_{1}\left(\frac{a}{d}, \frac{d}{s}\right) \quad \text { at } 0 \% \text { strain, } \\
\rho_{s}=\frac{V_{2}}{I} C_{2}\left(\frac{a}{d}, \frac{d}{s}\right) \quad \text { at } 0.04 \% \text { strain, }
\end{gathered}
$$

where $C_{1}=2.190560$ at $0 \%$ strain and $C_{2}=2.190314$ at $0.04 \%$ strain [47], $V_{1}$ and $V_{2}$ are the voltages across the inner probes at the $0 \%$ strain and $0.04 \%$ strain, respectively, and the input current is kept constant. When (12) is subtracted from (13), it gives (14)

$$
\Delta V=\rho_{s} I\left(\frac{1}{C_{2}}-\frac{1}{C_{1}}\right)=21 \mu V .
$$

It was shown that the change in voltage due to the change in dimension was small $(\sim 12 \%)$ compared to the change in resistivity $(\sim 88 \%)$.

Li et al. [48] observed the use of single-walled carbon nanotubes thin film strain sensors at the macroscale due to the electromechanical properties. The orientation of singlewalled carbon nanotubes affects the sensitivity of the film, that is, the electronic properties of randomly oriented singlewalled carbon nanotubes are not directionally dependent. The sensitivity to strains of aligned single-walled carbon nanotubes was expected to have higher sensitivity than that of randomly oriented single-walled carbon nanotubes. They demonstrated that single-walled carbon nanotube films were Raman active and can be used as a sensing material. A shift in the G-band modes of Raman spectra in a single-walled carbon nanotube film was observed when subjected to tensile load. A linear relationship between the tensile strain and shift in the G-band mode confirms that strain alters the electronic properties of the single-walled carbon nanotube film making it a potential strain sensor. Ramaratnam and Jalili [49] fabricated a piezoelectric carbon nanotube-based polyvinylidene fluoride (PVDF) polymer as a dynamic sensor for structural vibration control. The addition of carbon nanotubes significantly affects the electromechanical properties of the nanocomposites by increasing the sensing effectiveness of the piezoelectric polyvinylidene fluoride (PVDF) polymer. The use of single-walled carbon nanotube thin films showed a better response than multiwalled carbon nanotube thin films. Experimental and theoretical results show that increasing the Young's modulus of elasticity of the nanocomposites improves the sensing performance.

Kang et al. [20] reported the use of single-walled carbon nanotube thin films (buckypapers) and single-walled carbon nanotube-polymethylmethacrylate (PMMA) composites for strain sensing. The strain response of the buckypaper sensor showed higher sensitivity in the linear bending range. However, there was a saturated strain behavior over 500 microstrains under tension. This may be due to slippage among the carbon nanotubes bundles. During compression of the buckypaper sensor, there was no slippage of individual carbon nanotubes. This resulted in a lack of saturation as compared to the tension case. Though the buckypaper showed higher sensitivity, they may not be appropriate for measuring strain in the whole elastic range. Even though the composite sensors displayed less sensitivity than the buckypaper, they showed a linear symmetric strain response during compression and tension. This was because the bonding between the polymer and carbon nanotubes prevented slippage and effectively improved the strain in the composite sensor.

Li et al. [13] investigated the capability of using thin films of multiwalled carbon nanotubes as strain sensors. They used a uniaxial load/unload tensile test to estimate the sensing characteristics of the multiwalled carbon nanotube thin film. The results showed that a change in resistance was related to the applied strain as shown in Figure 13.

Pham et al. [50] studied the resistivity of multiwalled carbon nanotube polymer composite films under tensile strain and their promising application as strain sensors with tailored sensitivity. The electrical resistance of the nanocomposite film sensor was subjected to various tensile strains to evaluate the sensitivity of the film sensor. A semiempirical model, which depends on percolation theory, was used to relate the sensitivity factor and the applied strain. The conductive network density is a function of the sensitivity of the conductive film. It can be adjusted by controlling the nanotube loading, nanotube dispersion, and the fabrication process of the film. Vemuru et al. [15] used multiwalled carbon nanotubes as strain sensors. It was shown experimentally that a linear relationship exists between the change in voltage and change in strain. The efficiency of the film sensor in measuring strain at a macroscale was investigated and was very favorable indicating the use of multiwalled carbon nanotube thin films as a potential strain sensor at the macroscale. Ramaratnam and Jalili [49] demonstrated the use of multiwalled carbon 


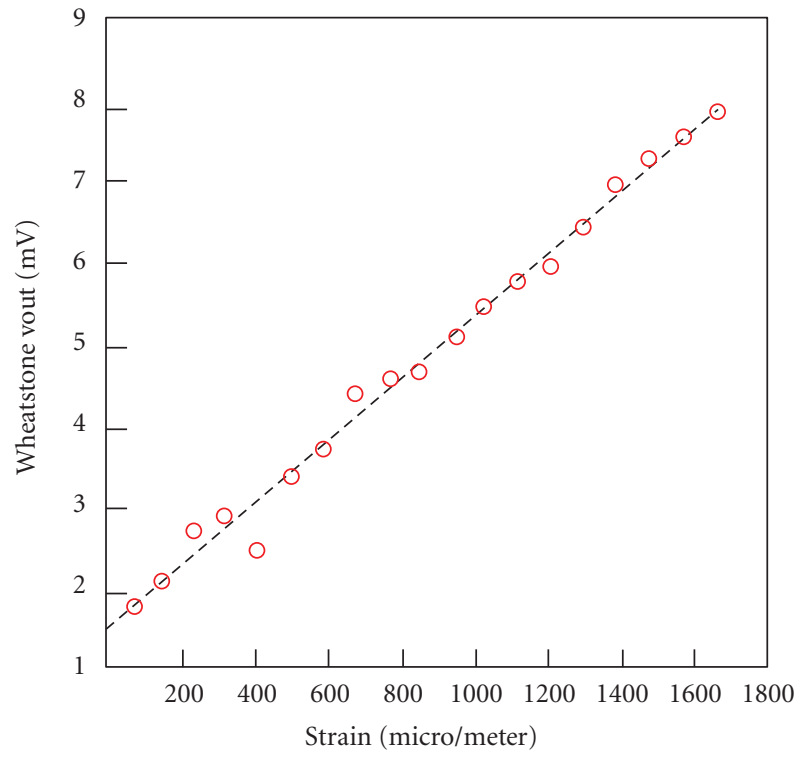

FIGURE 13: Multiwalled carbon nanotube thin film voltage output versus mechanical strain [13].

nanotube thin films as carbon nanotube/polycarbonate nanocomposite fillers. The thin film can act as a strain sensing material within the composites. An instant change was noticed in the resistivity $\left(\Delta R / R_{o}\right)$ of the nanocomposite when subjected to tensile strain with a gauge factor of about 3.5 times that of a conventional strain gauge. By improving mechanical properties like strength, stiffness, and structural damping, multiwalled carbon nanotube thin films can perform as multifunctional materials. Yin and others worked [51] on strain sensors made from an epoxy-based nanocomposites using multiwalled carbon nanotubes, that is, LMWNT-10. They detected that the piezoresitivity of the strain sensor was linear and antisymmetric when subjected to tensile and compressive strain. These properties were different from multiwalled carbon nanotubes, MWNT-7 [52, 53]. The difference in the piezoresistivity between the two types of sensors was probably due to the working mechanisms. For LMWNT-10, which has a smaller diameter and curved shapes, the principal working mechanism of the sensors is the piezoresistivity of multiwalled carbon nanotubes due to deformation. This resulted in a linear and antisymmetric piezoresistivity of the nanocomposite sensor. On the contrary, the main working mechanism of the strain sensor created from MWNT-7 with a larger diameter and straight shapes is the tunneling effect due to changes in distance among the multiwalled carbon nanotubes caused by applied strain.

Wang et al. [54] investigated the piezoresistivity of multiwalled carbon nanotube silicone rubber composites under uniaxial pressure. The experimental results showed that the active carboxyl radical on multiwalled carbon nanotubes can efficiently enhance the homogenous distribution and alignment of conductive paths in the composites. As a result, the composites presented positive piezoresistance with improved sensitivity and linearity for pressure, both of which are

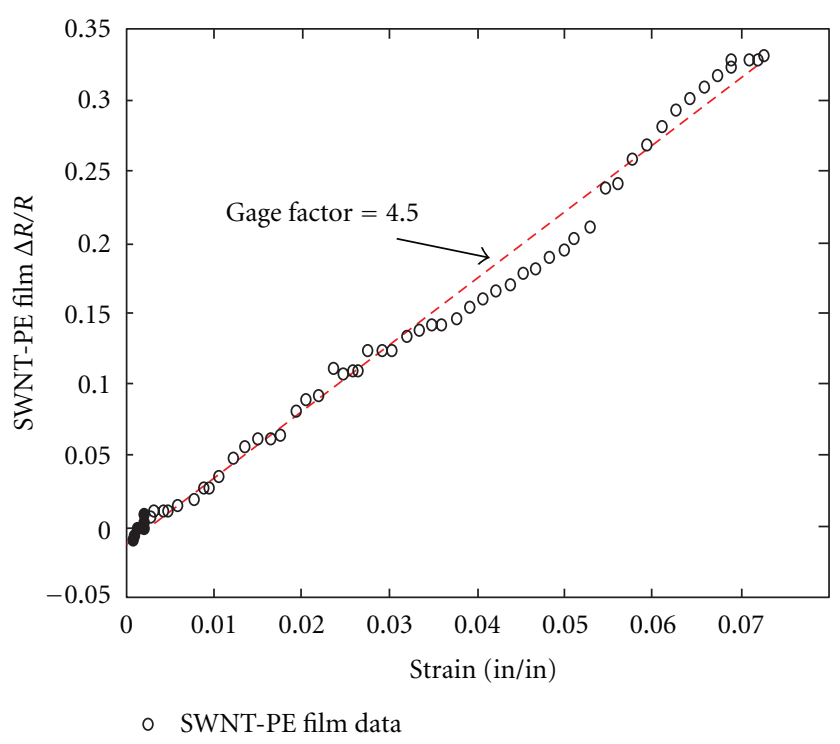

FIGURE 14: The change in resistivity versus strain of a single-walled carbon nanotube-polyelectrolyte thin film strain sensor [14].

important parameters for sensor applications. Kenneth et al. [14] used an easy and low-cost approach to manufacture single-walled carbon nanotube-polyelectrolyte composites by using a layer-by-layer assembly process (LBL). The electromechanical properties of the composite thin film sensors can be adjusted to have high sensitivity factors based on the initial LBL fabrication parameters, that is, type of carbon nanotubes, concentration of the single-walled carbon nanotubes, and the type of polyelectrolyte used. Monotonic tensile loading and four-point bending tests were used to estimate the sensitivity of a single-walled carbon nanotubepolyelectrolyte strain sensor mounted on a structural material as shown in Figure 14. With gauge factors greater than the traditional metal strain gauges, single-walled carbon nanotube-polyelectrolyte thin films serve as promising strain sensors for structural monitoring.

$\mathrm{Bu}$ et al. [17] analyzed the effect of sonication time on the sensitivity of carbon nanotube thin film strain sensors. The uniformity of the carbon nanotube thin films and the reproducibility of the resistance were considerably improved by sonication time. Multiwalled carbon nanotubes showed better sensing characteristics than single-walled carbon nanotubes with a strain up to $0.1 \%$. Two strain sensing regions were noticed for multiwalled carbon nanotubes probably due to variations in the response properties of contact resistance and intrinsic resistance to strain. For strain smaller than $0.1 \%$, the sensitivity of multiwalled carbon nanotubes was low, about 2.5 and for strain within $0.1 \%$ to $1 \%$, the sensitivity was about 5 with reproducible sensing characteristics. Strain sensing of single-walled carbon nanotube thin films was similar to that of multiwalled carbon nanotubes for strain under $0.2 \%$. The sensitivities were found to decrease for strain from $0.2 \%$ to $0.3 \%$; for higher strains from $0.5 \%$ to $1 \%$., the resistance changes greatly and without reproducibility. In a nutshell, multiwalled carbon nanotube thin 


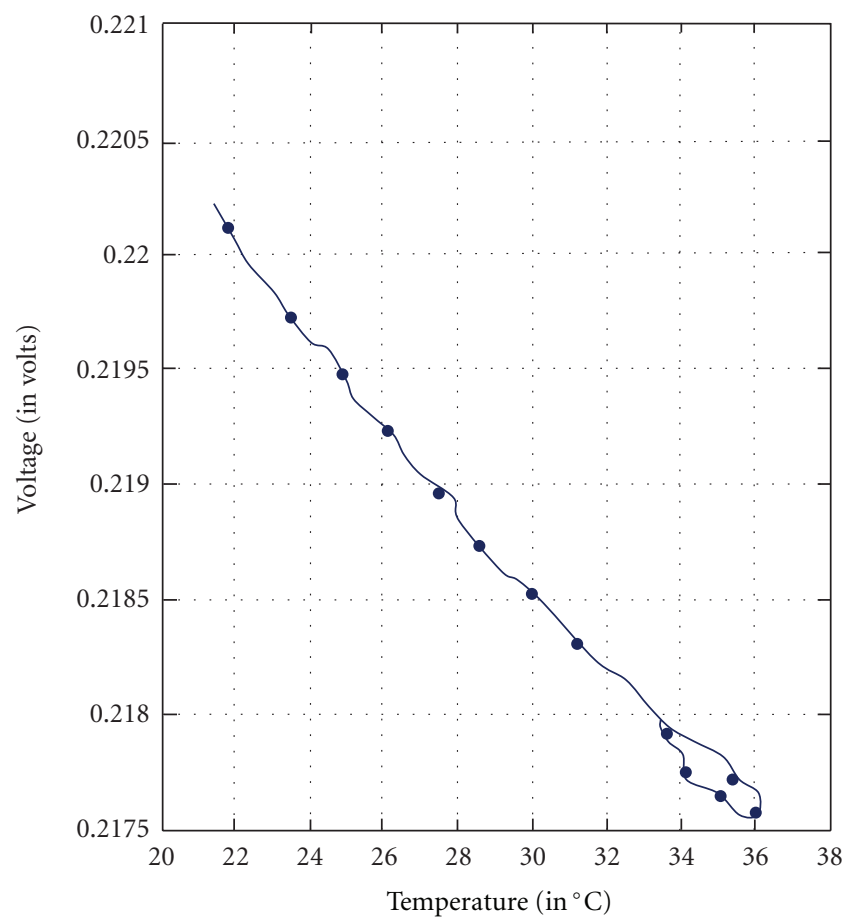

(a)

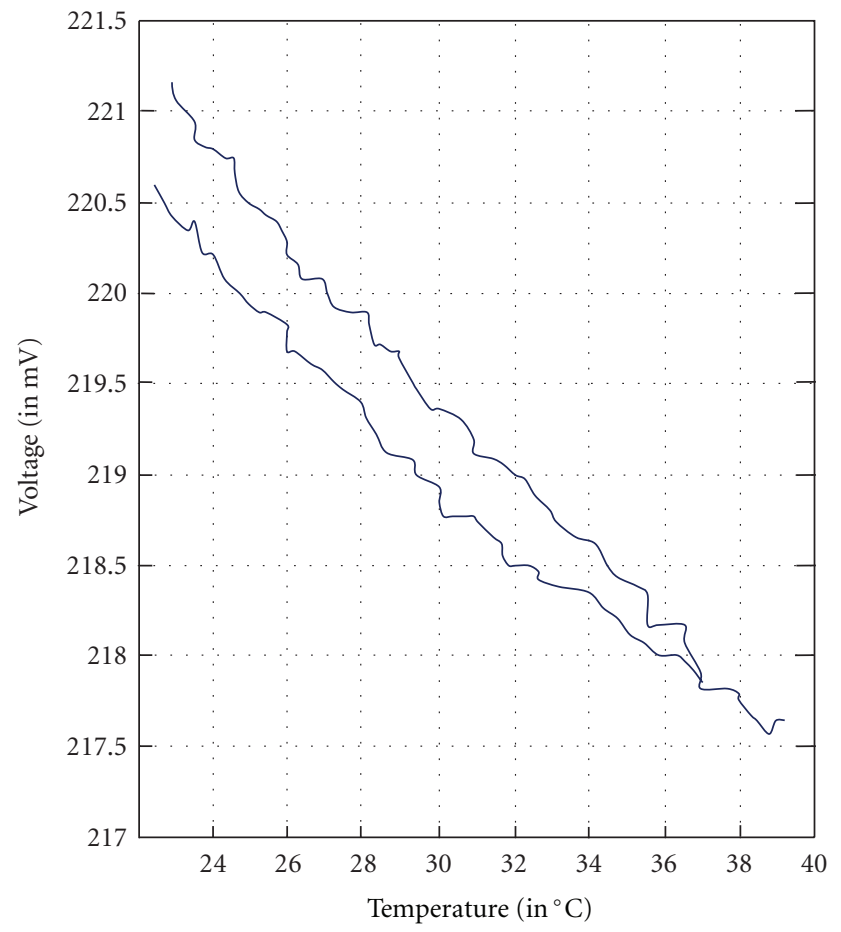

(b)

Figure 15: Voltage as a function of temperature as the brass specimen is heated: (a) from $21.1^{\circ} \mathrm{C}$ to $35^{\circ} \mathrm{C}$, (b) from $21.1^{\circ} \mathrm{C}$ to $37.8^{\circ} \mathrm{C}$ and cooled [15].

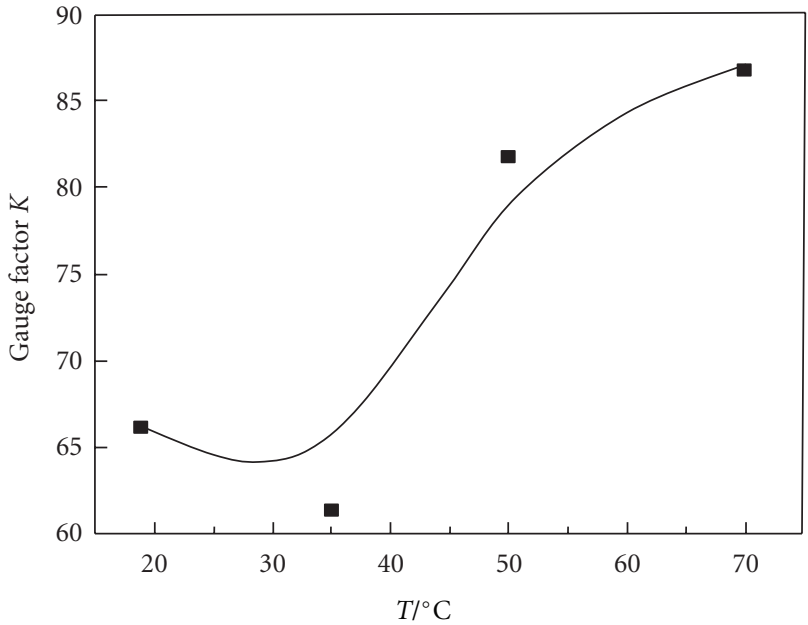

FIGURE 16: The gauge factor in carbon nanotube thin films less than 500 microstrains. Below $30^{\circ} \mathrm{C}$, the gauge factor decreased with increasing temperature, but increased above $30^{\circ} \mathrm{C}$. The gauge factor at $70^{\circ} \mathrm{C}$ is nearly one and a half times larger than at $19^{\circ} \mathrm{C}$ under 500 microstrain [16].

film sensors showed an exceptional sensing characteristic and a larger allowed working range than single-walled carbon nanotube thin films.

5.1. Factors Affecting Resistivity of Carbon Nanotube Thin Film Strain Sensors. Current research in developing carbon nanotube thin film strain sensors has achieved great improvement because of the advantage it has over traditional strain sensors. Carbon nanotube thin film strain sensors can be multidirectional, and they offer the potential of being embedded in composites. However, there are several limitations which must be overcome before carbon nanotube thin films can be used as sensing materials for real structures. Two of these problems are repeatability and stability. Repeatability can be explained as the repeatable resistance-strain performance, while stability is described as stable resistance. It has been shown that the resistance of carbon nanotube polymer strain sensors varies with or without strain $[20,55]$, which can lead to difficulties in practical situations. Resistance of carbon nanotube thin films varies due to a number of factors such as strain, defects, temperature, chemical effects, and size effects $[13,55]$. Carbon nanotube thin film sensors also display resistance hysteresis in a cyclic strain loading [56-58], which signifies an irreversible deterioration of carbon nanotube/polymer interfaces. Buckypaper has also been used as strain sensing materials $[13,45,50]$, but because of the low strength and permanent deformation after a strain of up $0.04 \%$, they cannot be used in applications that requires multifunctionality and repeatability.

Results obtained from experiments performed by Vemuru et al. [15] showed that there was a linear relationship between temperature and change in voltage. An increase in temperature resulted in an increase in the conductivity of the nanotube thin film as shown in Figure 15. 


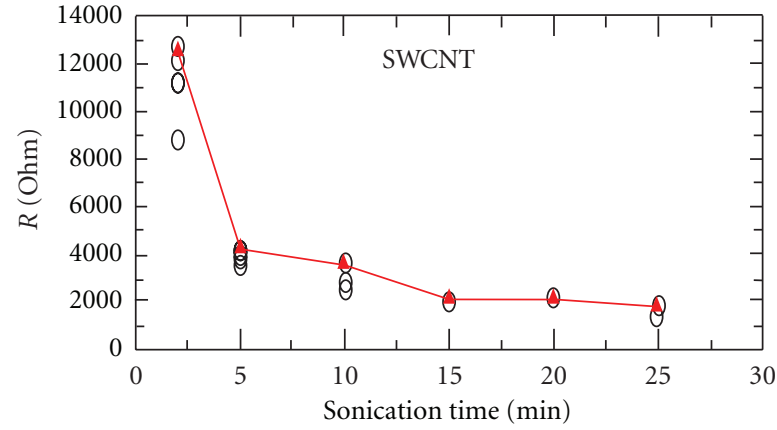

$0 \quad R_{i}$

$\triangle<R>$

(a)

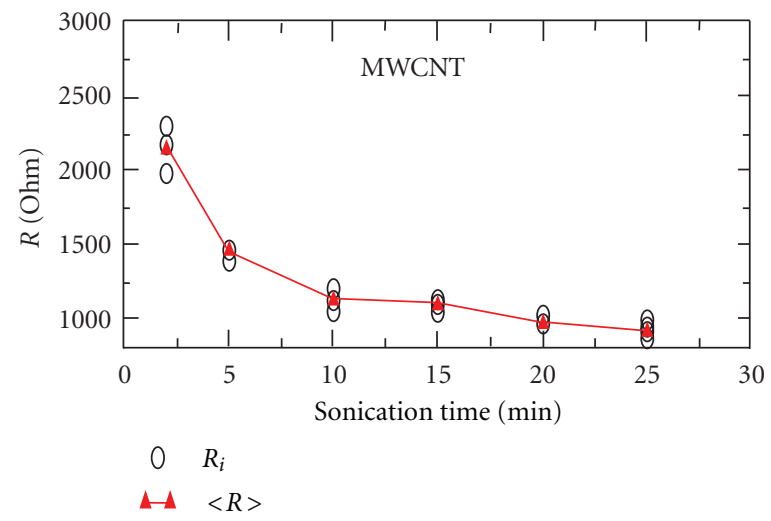

(c)

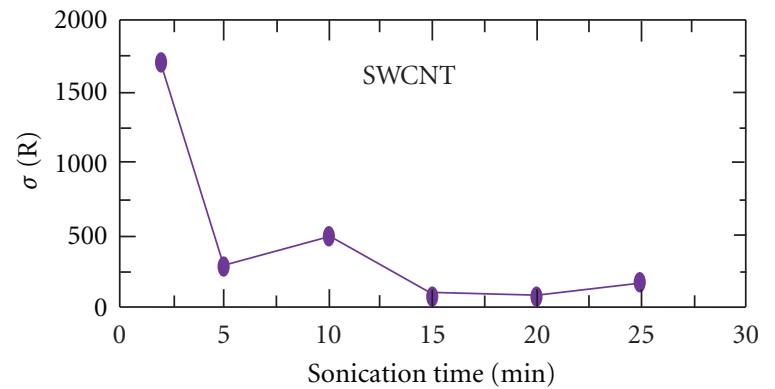

(b)

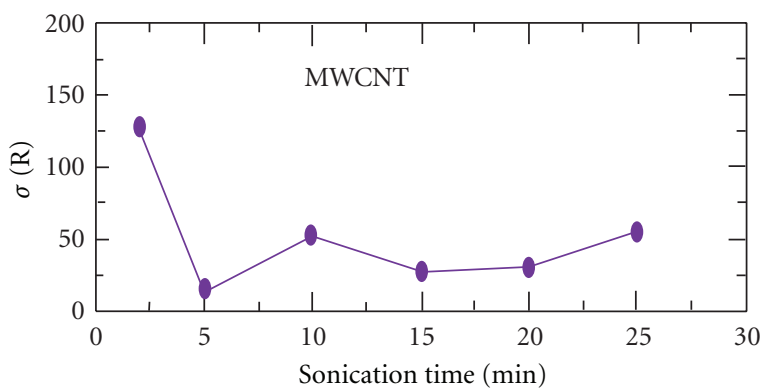

(d)

FIGURE 17: The resistance values of the specimens and their standard deviations versus sonication time. (a, b) Single-walled carbon nanotubes, (c, d) multiwalled carbon nanotubes [17].

Koratkar et al. [59] obtained results that were similar for a temperature range of $20-200^{\circ} \mathrm{C}$. The vertically aligned multiwalled carbon nanotubes show resistance as temperature dependent. Hone et al. [60] found that change in the electrical resistance of aligned films of single-walled carbon nanotubes is affected by temperature. They showed that the electric resistivity of magnetic field-aligned single-walled carbon nanotubes display anisotropic behavior with the alignment axis, while the thermal conductivity is the same when measured in a parallel and perpendicular direction to the axis. A relationship between the change in voltage and temperature was also studied by Bu et al. [17]. A negative temperature dependence of the carbon nanotube film resistance was noticed when cooled and heated for selected semiconducting carbon nanotubes in the temperature range from $20^{\circ} \mathrm{C}$ to $90^{\circ} \mathrm{C}$. The same result was also observed for unselected semiconducting and multiwalled carbon nanotubes, but with a lower temperature coefficient. Barberio et al. [16] studied the resistivity of multiwalled carbon nanotube samples at temperatures between $27^{\circ} \mathrm{C}$ and $1627^{\circ} \mathrm{C}$. They concluded that there was a monotonic decrease in the resistivity with an increase in temperature. The gauge factor of pristine carbon nanotube films increases with temperature as shown in Figure 16. (Error: Figure 16 is from [11]). The high gauge factors were probably due to different qualities of carbon nanotube films, for example pressure-induced changes in the band gap in pristine carbon nanotube films, defects, and measuring conditions [11]. (Error: Figure 16 is from [11]).

Other factors have shown significant effects on the electrical resistivity of carbon nanotubes, that is, exposure to different gases also affects the resistivity of the carbon nanotubes [61]. Processing parameters like sonication time and concentration of surfactant have been studied, along with their effect on the resistivity of carbon nanotubes [17].

The uniformity of the carbon nanotube films and reproducibility of the film's resistance are significantly improved by a suitable sonication time as shown in Figure 17.

\section{Conclusion}

Research areas related to carbon nanotubes have seen phenomenal development in recent years. An attempt has been made to provide the most contemporary overview possible of carbon nanotube-based strain sensors and their applications. The unique properties that make carbon nanotubes applicable as strain sensors have been reviewed. This paper signifies the type of carbon nanotubes best suited for strain sensors. It concludes that for carbon nanotube-based strain sensing applications, the small-gap semiconducting single-walled 
carbon nanotubes offer the greatest sensitivity. The capability of carbon nanotube thin films in measuring strain on the macroscale has been investigated. Results from experiments imply that there is a linear relationship between the measured change in resistance and strain. The temperature effect on the resistance was also investigated. The resistance was found to be temperature dependent for both single-walled and multiwalled carbon nanotubes.

Carbon nanotube films are composed of randomly oriented single-walled or multiwalled carbon nanotubes, which make their electronic properties independent of direction. Hence taking measurements along these different directions provides corresponding strains. Carbon nanotube films can also act as strong structural materials and sensors in composites. Such composites are of importance in smart structures.

The applications of carbon nanotubes for strain sensors will be explored in future work as the importance of nanotechnology research for strain sensing develops. Nevertheless, carbon nanotubes have yet to cross many technological obstacles in order to achieve their potential as the preferred material for strain sensor applications.

\section{References}

[1] S. Iijima, "Helical microtubules of graphitic carbon," Nature, vol. 354, no. 6348, pp. 56-58, 1991.

[2] A. Maiti, A. Svizhenko, and M. P. Anantram, "Electronic transport through carbon nanotubes: Effects of structural deformation and tube chirality," Physical Review Letters, vol. 88, no. 12, pp. 1268051-1268054, 2002.

[3] M. Dresselhaus, G. Dresselhaus, and P. Eklund, Science of Fullerenes and Carbon Nanotubes, Academic Press, San Diego, Calif, USA, 1996.

[4] R. Saito, G. Dresselhaus, and M. S. Dresselhaus, Physical Properties of Carbon Nanotubes, Imperial College Press, 1998.

[5] M. A. Cullinan and M. L. Culpepper, "Carbon nanotubes as piezoresistive microelectromechanical sensors: theory and experiment," Physical Review B, vol. 82, no. 11, Article ID 115428, 2010.

[6] T. W. Tombler, C. Zhou, L. Alexseyev et al., "Reversible electromechanical characteristics of carbon nanotubes under local-probe manipulation," Nature, vol. 405, no. 6788, pp. 769-772, 2000.

[7] E. D. Minot, Y. Yaish, V. Sazonova, J. Y. Park, M. Brink, and P. L. McEuen, "Tuning carbon nanotube band gaps with strain," Physical Review Letters, vol. 90, no. 15, pp. 156401/1-156401/4, 2003.

[8] R. J. Grow, Q. Wang, J. Cao, D. Wang, and H. Dai, "Piezoresistance of carbon nanotubes on deformable thin-film membranes," Applied Physics Letters, vol. 86, no. 9, Article ID 093104, 3 pages, 2005.

[9] J. Cao, Q. Wang, and H. Dai, "Electromechanical properties of metallic, quasimetallic, and semiconducting carbon nanotubes under stretching," Physical Review Letters, vol. 90, no. 15, pp. 157601/1-157601/4, 2003.

[10] G. T. Pham, Characterization and Modeling of Piezo-Resistive Properties of Carbon Nanotube-Based Conductive Polymer Composites, Industrial \& Manufacturing Engineering Department, College of Engineering, Florida State University, Tallahassee, FL, USA, 2008.
[11] Y. Li, W. Wang, K. Liao, C. Hu, Z. Huang, and Q. Feng, "Piezoresistive effect in carbon nanotube films," Chinese Science Bulletin, vol. 48, no. 2, pp. 125-127, 2003.

[12] W. L. Wang, K. J. Liao, Y. Li, and Y. T. Wang, "Piezoresistive effect of doped carbon nanotube/cellulose films," Chinese Physics Letters, vol. 20, no. 9, pp. 1544-1547, 2003.

[13] X. Li, C. Levy, and L. Elaadil, "Multiwalled carbon nanotube film for strain sensing," Nanotechnology, vol. 19, no. 4, Article ID 045501, 2008.

[14] J. L. Kenneth, P. L. Jerome, and A. K. Nicholas, "Conformable single-walled carbon nanotube thin film," in Proceedings of the 5th International Workshop on Structural Health Monitoring, Stanford, Calif, USA, 2005.

[15] S. M. Vemuru, R. Wahi, S. Nagarajaiah, and P. M. Ajayan, "Strain sensing using a multiwalled carbon nanotube film," Journal of Strain Analysis for Engineering Design, vol. 44, no. 7, pp. 555-562, 2009.

[16] M. Barberio, M. Camarca, P. Barone, A. Bonanno, A. Oliva, and $\mathrm{F} . \mathrm{Xu}$, "Electric resistivity of multi-walled carbon nanotubes at high temperatures," Surface Science, vol. 601, no. 13, pp. 2814-2818, 2007.

[17] L. Bu, J. Steitz, and O. Kanoun, "Influence of processing parameters on properties of strain sensors based on carbon nanotube films," in Proceedings of the 7th International Multi-Conference on Systems, Signals and Devices (SSD '10), June 2010.

[18] H. Kusumi, K. F. H. Saito, and K. Narita, "Monitoring system of tension strain on rock slope by optical strain sensor," Rock mechanics, vol. 2001, pp. 175-180, 2001.

[19] M. Kreuzer, Strain Measurement with Fiber Bragg Grating Sensors, HBM, Darmstadt, Germany.

[20] I. Kang, M. J. Schulz, J. H. Kim, V. Shanov, and D. Shi, "A carbon nanotube strain sensor for structural health monitoring," Smart Materials and Structures, vol. 15, no. 3, pp. 737-748, 2006.

[21] G. Overney, W. Zhong, and D. Tománek, "Structural rigidity and low frequency vibrational modes of long carbon tubules," Zeitschrift für Physik D, vol. 27, no. 1, pp. 93-96, 1993.

[22] B. I. Yakobson, C. J. Brabec, and J. Bernholc, "Nanomechanics of carbon tubes: instabilities beyond linear response," Physical Review Letters, vol. 76, no. 14, pp. 2511-2514, 1996.

[23] E. W. Wong, P. E. Sheehan, and C. M. Lieber, "Nanobeam mechanics: elasticity, strength, and toughness of nanorods and nanotubes," Science, vol. 277, no. 5334, pp. 1971-1975, 1997.

[24] B. G. Demczyk, Y. M. Wang, J. Cumings et al., "Direct mechanical measurement of the tensile strength and elastic modulus of multiwalled carbon nanotubes," Microscopy and Microanalysis, vol. 12, no. 2, pp. 934-935, 2006.

[25] R. Andrews, D. Jacques, A. M. Rao et al., "Nanotube composite carbon fibers," Applied Physics Letters, vol. 75, no. 9, pp. 13291331, 1999.

[26] S. Frank, P. Poncharal, Z. L. Wang, and W. A. De Heer, "Carbon nanotube quantum resistors," Science, vol. 280, no. 5370, pp. 1744-1746, 1998.

[27] A. Bachtold, C. Strunk, J. P. Salvetat et al., "Aharonov-Bohm oscillations in carbon nanotubes," Nature, vol. 397, no. 6721, pp. 673-675, 1999.

[28] L. Yang, M. P. Anantram, J. Han, and J. P. Lu, "Band-gap change of carbon nanotubes: effect of small uniaxial and torsional strain," Physical Review B, vol. 60, no. 19, pp. 1387413878, 1999.

[29] A. Kleiner and S. Eggert, "Band gaps of primary metallic carbon nanotubes," Physical Review B, vol. 63, no. 7, Article ID 073408, 4 pages, 2001. 
[30] S. Reich, C. Thomsen, and J. Maulsch, Carbon Nanotubes: Basic Concepts and Physical Properties, Wiley-VCH, New York, NY, USA, 2004.

[31] M. A. Cullinan and M. L. Culpepper, "Carbon nanotubes as piezoresistive microelectromechanical sensors: theory and experiment," Physical Review B, vol. 82, no. 11, Article ID $115428,2010$.

[32] C. Stampfer, A. Jungen, R. Linderman, D. Obergfell, S. Roth, and C. Hierold, "Nano-electromechanical displacement sensing based on single-walled carbon nanotubes," Nano Letters, vol. 6, no. 7, pp. 1449-1453, 2006.

[33] G. T. A. Kovacs, Micromachined Transducers Sourcebook, WCB; McGraw-Hill, Boston, Mass, USA, 1998.

[34] C. L. Kane and E. J. Mele, "Size, shape, and low energy electronic structure of carbon nanotubes," Physical Review Letters, vol. 78, no. 10, pp. 1932-1935, 1997.

[35] T. A. Gloor and F. Mila, "Strain induced correlation gaps in carbon nanotubes," European Physical Journal B, vol. 38, no. 1, pp. 9-12, 2004.

[36] L. Liu, C. S. Jayanthi, M. Tang et al., "Controllable reversibility of an $\mathrm{sp}^{2}$ to $\mathrm{sp}^{3}$ transition of a single wall nanotube under the manipulation of an AFM tip: a nanoscale electromechanical switch?” Physical Review Letters, vol. 84, no. 21, pp. 4950-4953, 2000.

[37] W. Liang, M. Bockrath, D. Bozovic, J. H. Hafner, M. Tinkham, and H. Park, "Fabry-Perot interference in a nanotube electron waveguide," Nature, vol. 411, no. 6838, pp. 665-669, 2001.

[38] C. Berger, Y. Yi, Z. L. Wang, and W. A. De Heer, "Multiwalled carbon nanotubes are ballistic conductors at room temperature," Applied Physics A, vol. 74, no. 3, pp. 363-365, 2002.

[39] M. Zamkov, A. S. Alnaser, B. Shan, Z. Chang, and P. Richard, "Probing the intrinsic conductivity of multiwalled carbon nanotubes," Applied Physics Letters, vol. 89, no. 9, Article ID 093111, 3 pages, 2006.

[40] R. Heyd, A. Charlier, and E. McRae, "Uniaxial-stress effects on the electronic properties of carbon nanotubes," Physical Review B, vol. 55, no. 11, pp. 6820-6824, 1997.

[41] L. Yang and J. Han, "Electronic structure of deformed carbon nanotubes," Physical Review Letters, vol. 85, no. 1, pp. 154-157, 2000.

[42] J. G. Simmons, "Generalized formula for the electric tunnel effect between similar electrodes separated by a thin insulating film," Journal of Applied Physics, vol. 34, no. 6, pp. 1793-1803, 1963.

[43] P. Sheng, E. K. Sichel, and J. I. Gittleman, "Fluctuationinduced tunneling conduction in carbon-polyvinylchloride composites," Physical Review Letters, vol. 40, no. 18, pp. 11971200, 1978.

[44] Q. Cao and J. A. Rogers, "Ultrathin films of single-walled carbon nanotubes for electronics and sensors: a review of fundamental and applied aspects," Advanced Materials, vol. 21, no. 1, pp. 29-53, 2009.

[45] P. Dharap, Z. Li, S. Nagarajaiah, and E. V. Barrera, "Nanotube film based on single-wall carbon nanotubes for strain sensing," Nanotechnology, vol. 15, no. 3, pp. 379-382, 2004.

[46] O. Zhou, R. M. Fleming, D. W. Murphy et al., "Defects in carbon nanostructures," Science, vol. 263, no. 5154, pp. 1744 1747, 1994.

[47] I. Kang, M. J. Schulz, J. H. Kim, V. Shanov, and D. Shi, "A carbon nanotube strain sensor for structural health monitoring," Smart Materials and Structures, vol. 15, no. 3, pp. 737-748, 2006.
[48] Z. Li, P. Dharap, S. Nagarajaiah, E. V. Barrera, and J. D. Kim, "Carbon nanotube film sensors," Advanced Materials, vol. 16, no. 7, pp. 640-577, 2004.

[49] A. Ramaratnam and N. Jalili, "Reinforcement of piezoelectric polymers with carbon nanotubes: pathway to next-generation sensors," Journal of Intelligent Material Systems and Structures, vol. 17, no. 3, pp. 199-208, 2006.

[50] G. T. Pham, A. Colombo, Y. B. Park, C. Zhang, and B. Wang, "Nanotailored thermoplastic/carbon nanotube composite strain sensor," in Proceedings of the Multifunctional Nanocomposites International Conference, pp. 277-283, September 2006.

[51] G. Yin, N. Hu, Y. Karube, Y. Liu, Y. Li, and H. Fukunaga, "A carbon nanotube/polymer strain sensor with linear and antisymmetric piezoresistivity," Journal of Composite Materials, vol. 45, no. 12, pp. 1315-1323, 2011.

[52] N. Hu, Y. Karube, C. Yan, Z. Masuda, and H. Fukunaga, "Tunneling effect in a polymer/carbon nanotube nanocomposite strain sensor," Acta Materialia, vol. 56, no. 13, pp. 2929-2936, 2008.

[53] N. Hu, Y. Karube, M. Arai et al., "Investigation on sensitivity of a polymer/carbon nanotube composite strain sensor," Carbon, vol. 48 , no. 3 , pp. 680-687, 2010.

[54] P. Wang, S. Geng, and T. Ding, "Effects of carboxyl radical on electrical resistance of multi-walled carbon nanotube filled silicone rubber composite under pressure," Composites Science and Technology, vol. 70, no. 10, pp. 1571-1573, 2010.

[55] K. J. Loh, J. Kim, J. P. Lynch, N. W. S. Kam, and N. A. Kotov, "Multifunctional layer-by-layer carbon nanotube-polyelectrolyte thin films for strain and corrosion sensing," Smart Materials and Structures, vol. 16, no. 2, pp. 429-438, 2007.

[56] L. Böger, M. H. G. Wichmann, L. O. Meyer, and K. Schulte, "Load and health monitoring in glass fibre reinforced composites with an electrically conductive nanocomposite epoxy matrix," Composites Science and Technology, vol. 68, no. 7-8, pp. 1886-1894, 2008.

[57] E. T. Thostenson and T. W. Chou, "Real-time in situ sensing of damage evolution in advanced fiber composites using carbon nanotube networks," Nanotechnology, vol. 19, no. 21, Article ID 215713, 2008.

[58] M. Knite, V. Tupureina, A. Fuith, J. Zavickis, and V. Teteris, "Polyisoprene-multi-wall carbon nanotube composites for sensing strain," Materials Science and Engineering C, vol. 27, no. 5-8, pp. 1125-1128, 2007.

[59] N. Koratkar, A. Modi, E. Lass, and P. Ajayan, "Temperature effects on resistance of aligned multiwalled carbon nanotube films," Journal of Nanoscience and Nanotechnology, vol. 4, no. 7, pp. 744-748, 2004.

[60] J. Hone, M. C. Llaguno, N. M. Nemes et al., "Electrical and thermal transport properties of magnetically aligned single wall carbon nanotube films," Applied Physics Letters, vol. 77, no. 5, pp. 666-668, 2000.

[61] P. G. Collins and P. Avouris, "Nanotubes for electronics," Scientific American, vol. 283, no. 6, pp. 62-69, 2000. 

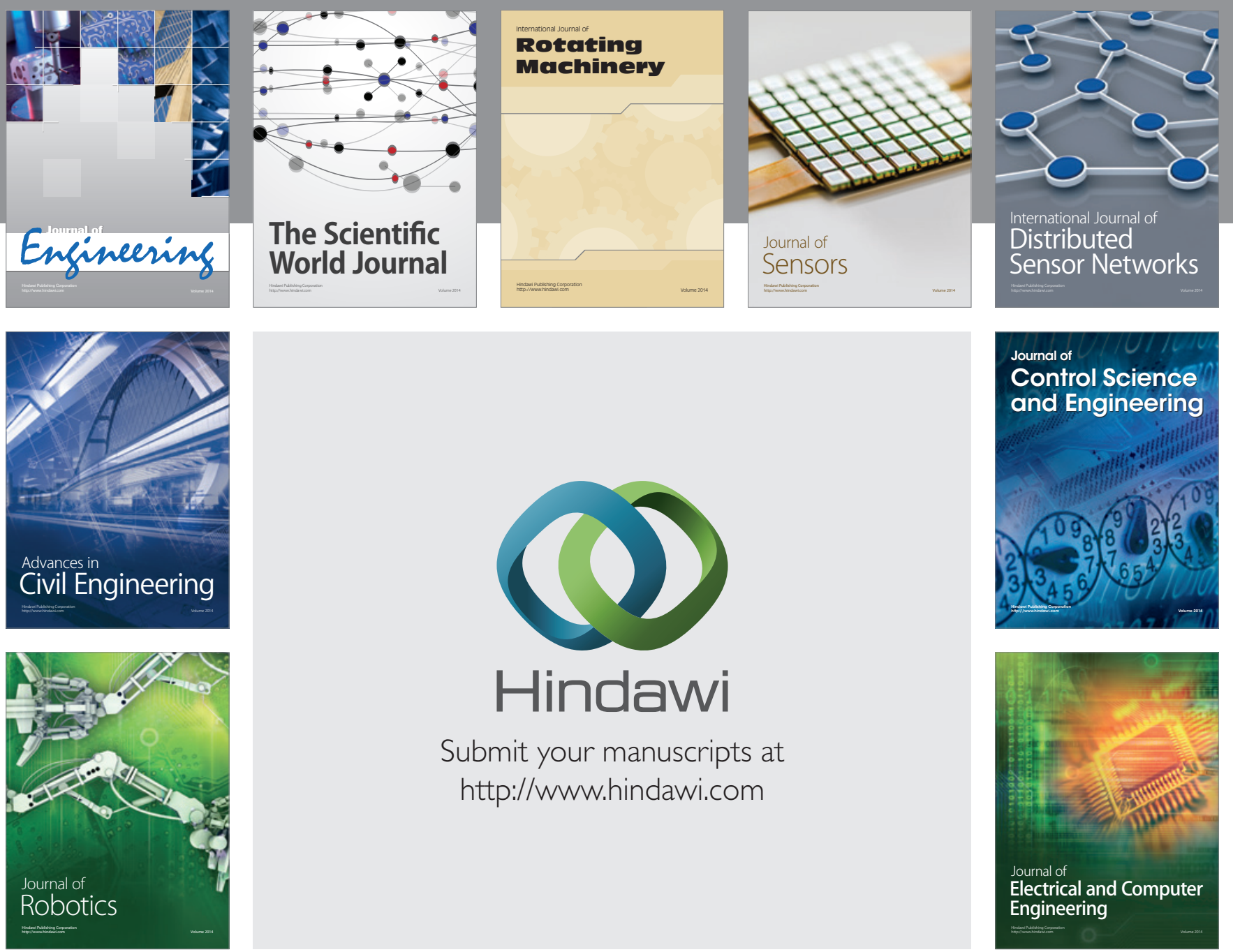

Submit your manuscripts at

http://www.hindawi.com
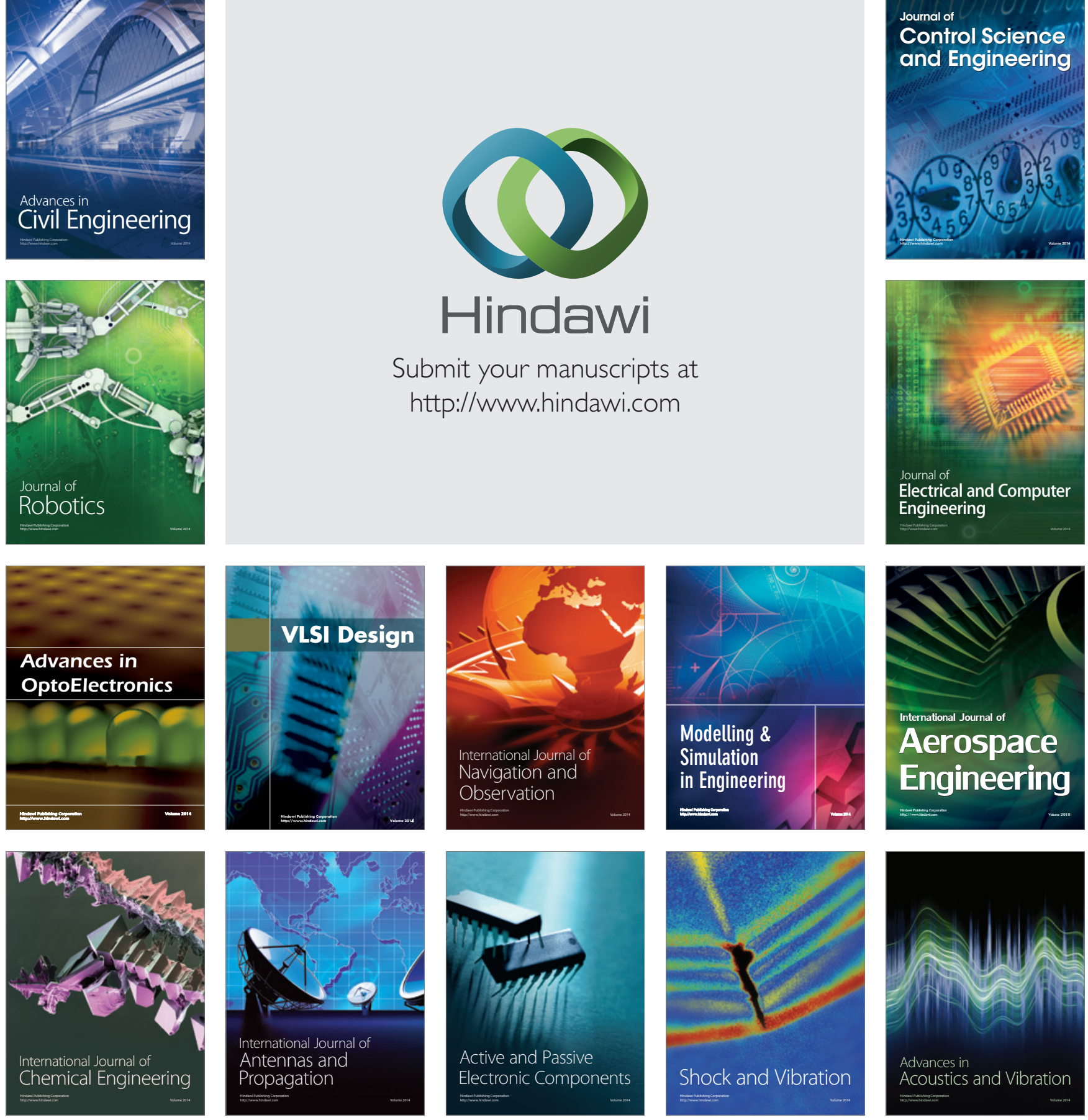